\title{
INTERFERENCE MEASUREMENTS OF WAVE LENGTHS IN THE IRON SPECTRUM (3233A-6750A)
}

\author{
By Keivin Burns, W. F. Meggers and Paul W. Merrill
}

\section{CONTENTS}

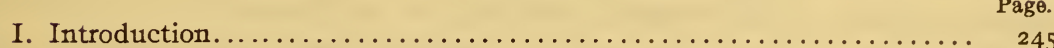

II. The region of the spectrum covered...................... 246

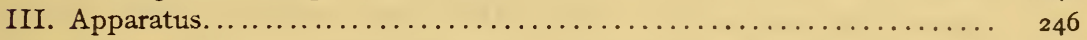

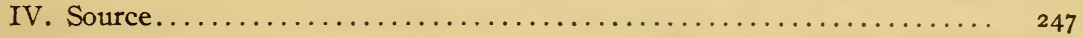

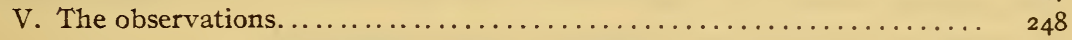

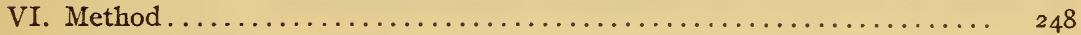

I. Standards and corrections....................... 248

2. Use of three interferometers....................... 249

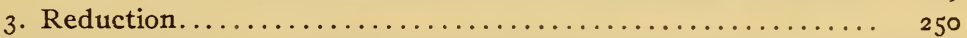

VII. Results....................................... 250

I. Use of earlier measurements....................... 250

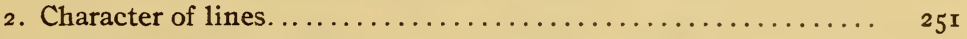

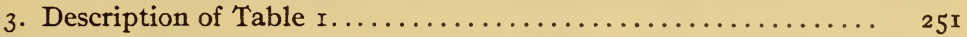

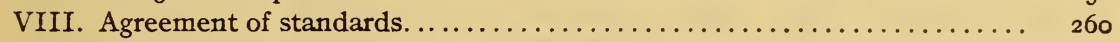

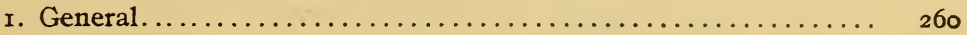

2. Standards for which B. S. minus I. A. is greater than $0.00 \mathrm{~A} \ldots 260$

3. Unsatisfactory standards.......................... $26 \mathrm{r}$

4. Pole e̊ffect and interference measurements............ 26r

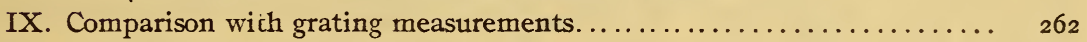

I. General agreement............................... 262

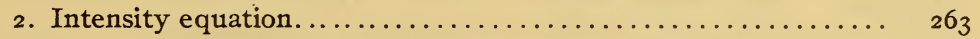

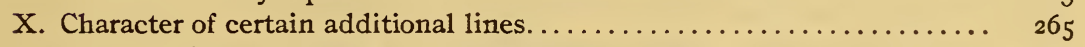

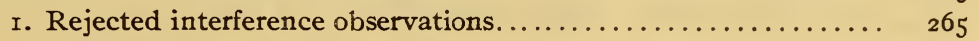

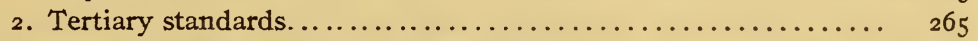

XI. Correlation of sharpness with other properties............... 267

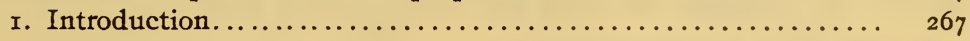

2. Pole effect. ................................... ${ }_{268}$

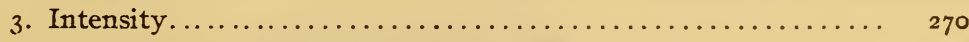

4. Behavior under pressure........................ $270 . \ldots \ldots \ldots \ldots$

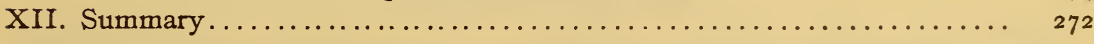

\section{INTRODUCTION}

The Bureau of Standards took up the matter of standard wave lengths of light over a year ago and a list of such wave lengths was published. ${ }^{1}$ In that paper attention was called to the need 
and demand for such work on account of its importance to astronomers, metrologists, physicists, and chemists. It may be stated that the demand for more accurate wave lengths is increasing. This demand is being made by the theoretical physicist and also by the analytical chemist. Some instances have come to the attention of the Bureau in the past year in which it was difficult or impossible to make an analysis, due to the present imperfect state of wave-length determinations. The first step must be to determine the standards of wave length and it is the purpose of this Bureau to make such determinations as accurately as possible throughout the entire length of spectrum which can be photographed. The present article is a continuation of the work published in the paper to which reference was made above. In that paper the reason for using the iron spectrum was given, the present state of standards was discussed, and the hope of cooperation with other observers was expressed.

\section{THE REGION OF THE SPECTRUM COVERED}

The present paper covers the region $3233 \mathrm{~A}$ to $675 \mathrm{OA}$ ( $\mathrm{IA}=\mathrm{I} \times$ $1 \mathrm{O}^{-7} \mathrm{~mm}$ ), the whole range of the international standards. There are too few of these standards for many purposes, so, wherever it was possible, lines were observed at intervals of about IoA; that is, the wave length of each successive line is IoA greater than that of the line preceding. At many points in the iron spectrum it is impossible to find sharp lines of sufficient intensity at such close intervals. In some cases, lines of an unsatisfactory character were measured in these regions and experience may prove that these lines are worthless as standards. No impurity lines were observed. The advisability of using impurity lines to fill gaps in the iron spectrum will be taken up in a later publication. At present we are of the opinion that these lines should be used with circumspection.

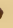

\section{APPARATUS}

The apparatus used was similar to that previously described. ${ }^{2}$ The light from the arc was focused on the interferometer by means of a simple quartz lens. The interferometer plates were quartz disks $8 \mathrm{~mm}$ in thickness, whose inner surfaces had a semitransparent coating of platinum, cathodically deposited. These plates were kept parallel to each other by means of invar sepa- 
rators. The aperture of the interferometer was limited to $9 \mathrm{~mm}$ by a diaphragm. The interference rings were projected upon the slit either by means of a quartz-fluorite lens or by a glass lens which was achromatic throughout the visible spectrum. This latter was designed by Mr. Tillyer and made at the Bureau of Standards. The spectrum was formed by an Anderson concave grating mounted in parallel light. The first order is very bright on one side, and the second on the same side is quite satisfactory in this respect. In the second order the scale is $5 \mathrm{~A}$ per millimeter; about $900 \mathrm{~A}$ can be photographed in sharp focus in the second order, and $1800 \mathrm{~A}$ in the first. The grating was ruled 299 lines per millimeter, there being 39800 lines in all. Light passing through the slit fell upon a concave mirror, from which it was returned as a parallel beam to the grating placed close beside the slit. The grating was mounted on one end of a webbed steel beam which could be rotated around the axis passing through the center of the grating. The other end of the beam slipped along a double track, thus bringing the different orders of the spectrum to the camera, which was free to slide lengthwise of the beam. When the proper region was reached the camera was clamped firmly to the double track, which was strongly cemented to a solid brick pier. The whole apparatus could be clamped to massive piers, which were all cemented to a thick concrete floor. The stability of the spectroscope apparently leaves nothing to be desired, as the longest exposures were perfectly sharp. On account of the fact that the slit and the center of the grating are $4^{\circ}$ apart, as seen from the mirror, there is a slight astigmatism in the image. The focus of the camera was usually set to make the rings sharp. In the first order region, $4900 \mathrm{~A}$ to $6800 \mathrm{~A}$, this procedure broadened the lines too much and a mean focus was used. The peculiar bend in the reduction curve in this region may be connected with this fact.

\section{SOURCE}

The source was an arc either of electrolytic iron or of Norway iron, the former being used for at least one plate in each region. The normal conditions recommended by the international wave length committee ${ }^{3}$ were rigorously fulfilled. The region $3500 \mathrm{~A}$ to $4200 \mathrm{~A}$ was observed with both 4 and 6 amperes, but no differences in wave length were detected as a result of the increased current strength. 


\section{THE OBSERVATIONS}

Seed 27 plates were used throughout, but to photograph the region $4900 \mathrm{~A}$ to $675^{\circ} \mathrm{A}$ the plates were bathed in pinaverdol and dicyanin with ammonia. In the region $3200 \mathrm{~A}$ to $4200 \mathrm{~A}$ there were measured: Four exposures of $\mathrm{I}$; five of 6 ; one each of $\mathrm{I}_{5}$, 20 , and 30 ; and two of 60 minutes' duration. In the region $3500 \mathrm{~A}$ to $4500 \mathrm{~A}$ the exposures were: Five of 2 ; five of 5 ; two of 20 ; and three of 25 minutes. The region 4 IooA to 5 I00A was covered by exposures as follows: Three of 3 ; two of 5 ; one of Io; four of 15 ; one of 25 ; and four of 50 minutes. Finally, in the region $4900 \mathrm{~A}$ to $6800 \mathrm{~A}$, the following exposures were measured: One of 1 ; two of 3 ; three of 5 ; three of 9 ; two of 15 ; three of 20 ; two of 90 ; and one each of 65,80 , and roo minutes' duration. The first three regions were photographed in the second order; $4900 \mathrm{~A}$ to $6800 \mathrm{~A}$ was taken in the first order. In almost every case two or more exposures were measured on the same plate.

These exposures were made without disturbing the interferometer. In 3 cases out of 24 there was a change in the thickness of about one part in a million, and in a few other cases there was a barely perceptible change. These changes were probably due to a variation in the temperature, although the thermometer beside the interferometer registered no appreciable change. By allowing the light to fall upon the interferometer for half an hour before taking the plate this trouble could be avoided. A short exposure was always made first, and another short exposure usually followed the long one. In any case, each exposure was worked out by itself and this expansion of the interferometer caused no difficulty. In a few cases the long exposure on a plate was too dense to permit of accurate measurement of a sufficient number of standards. In such cases the long and short exposures were tied together by lines of intermediate intensity. It will be seen at once that this method would lead to erroneous results in case there were an intensity equation. The question will be discussed later in connection with the grating observations.

\section{METHOD}

\section{STANDARDS AND CORRECTIONS}

The method of reduction was that of Buisson and Fabry, ${ }^{4}$ outlined in the former paper on wave lengths. Since the secondary I. A. standards were used, it is probable that at all points in the 
spectrum the resulting system is more nearly correct than would have been the case if the fundamental standard had been used. For these standards are the result of three independent series of observations, and in the mean are no doubt very nearly correct. The use of the fundamental standard would have introduced the troublesome question of phase change, and the results would surely have been no more nearly correct than any one of the three original series of observations. Having used the I. A. normals, it is probable that the mean wave length of several lines near any point in the spectrum is very nearly as accurate as the I. A. system at that point. Since the standards were distributed throughout the whole region, no correction to normal temperature and pressure was necessary.

\section{USE OF THREE INTERFEROMETERS}

In one particular the present investigation departs from the practices of former observers, namely, in the use of at least three interferometers for the observation of each line. This procedure has many advantages. For one thing, some lines can not be observed with a very high order of interference, and in order to measure the wave lengths of these lines one is tempted to use a low order, and so sacrifice the accuracy that might be obtained for other lines in using a thicker interferometer. By using a low, an intermediate, and a high order these poor lines may be measured with some accuracy, and the accuracy of the good lines is no doubt somewhat increased. The wave lengths of the sharp lines were not derived by means of the thickest interferometer alone for the reason that it was considered more important to eliminate the effect of a small error in the determination of the angular diameter of the ring system. The elimination of this error is accomplished by using more than one etalon, since the standards are situated throughout the whole region under observation. In changing from one interferometer to another, care was taken to rotate the plates with respect to each other in order to minimize the effect of slight irregularities in their surfaces. In the case of double lines, the components may be nearly in phase in one interferometer but out of step in another. In this way a few doubles were eliminated. A good illustration is found in $5269.5 \mathrm{~A}$. This line gives sharp interference of orders 38 thousand and 57 thousand, but shows two sets of rings of order 28 thousand. The wave lengths of the two components are 5269.495 and 
5269.595, the interval being great enough to be seen with a large grating. In a few other cases the wave length determined by one interferometer differed too much from that found by means of another, and the line was discarded on the ground that it might be a close double. Poor lines, of course, could not be observed with the higher orders of interference, and no line which is measured by means of only one interferometer is recommended as a standard where the highest accuracy is involved.

\section{REDUCTION}

Having I 5 to 30 standards on each plate, the thickness of the interferometer was determined from three or more of them and all wave lengths computed. Then all available standards (observed minus I. A.) were represented as closèly as possible by a smooth curve. The curve was usually a straight line excepting in the region $4900 \mathrm{~A}-6800 \mathrm{~A}$. In this latter region the line was bent at $6100 \mathrm{~A}$, and was always straight from $6100 \mathrm{~A}$ to $6800 \mathrm{~A}$. This bend is thought to be due to instrumental causes, yet it may be due in part to inaccuracies in the secondary standards. Having drawn the curve, all wave lengths were corrected by means of it.

\section{RESULTS}

\section{USE OF EARLIER MEASUREMENTS}

In the region $3233 \mathrm{~A}$ to $3600 \mathrm{~A}$ all wave lengths which had been published by the Bureau ${ }^{5}$ were remeasured by means of at least one interferometer whose thickness differed from that used in the earlier work. If the new wave length did not exactly check the old, the line was completely remeasured. All lines which were poorly determined (marked "C") in the earlier work were completely remeasured; this includes all lines between $3600 \mathrm{~A}$ and $370 r \mathrm{~A}$. The mean of all observations, both the earlier determinations and those made by the writers, was used to form the value found in Table I. From $5434 \mathrm{~A}$ to $675 \mathrm{OA}$ all lines, formerly measured by Burns, ${ }^{6}$ were completely remeasured unless they were too faint on the Bureau plates. Usually the mean of all observations, the early measurements by Burns and those by the writers, was used as the value to be published in Table $\mathrm{r}$. 
However, in some cases the dispersion used by Burns was considered inadequate to separate close pairs sufficiently, and only the observations made at the Bureau were used.

\section{CHARACTER OF LINES}

The limiting order with which a line can be observed is a direct measure of its sharpmess. Further, it is of interest to know that a line has been examined by means of more than one interferometer, as a double may show interference of a high order. Frequently a line will show poor rings in each of several orders, but usually, if a line is poor at a given order, it shows no rings at all when the path difference is increased 30 to 50 per cent. The character of the lines may be judged by an inspection of their behavior under increasing order, the data being found in Table $\mathrm{r}$. Unless otherwise noted all lines of wave length less than $3960 \mathrm{~A}$ which are included in the table showed sharp rings in interferometers of $2 \mathrm{~mm}$ and $3 \mathrm{~mm}$ thickness.

\section{DESCRIPTION OF TABLE 1}

Column I contains the observed wave length at $15^{\circ}$ and 760 $\mathrm{mm}$. Column 2 contains the intensity and character of the line as seen by means of a grating, using 6 or 7 amperes. " $b$ " $=$ broad; "d"=double; " $h "=$ hazy; " $1 "=$ shaded to red; " $r "=$ narrow reversed; " $\mathrm{v}$ " = shaded to violet. Column 3 contains letters which indicate the probable error deduced from the agreement of individual exposures. " $\mathrm{A}$ " indicates a probable error less than $0.0007 \mathrm{~A}$; " $\mathrm{B}$," $0.0007 \mathrm{~A}$ to $0.0012 \mathrm{~A}$; "C" means that the determination was poor. Column 4 contains the orders of interference in thousands which were used in measuring the wave length, and column 5 contains additional orders at which the lines were examined but not measured. An asterisk (*) indicates that the rings were measurable but not sharp; an exclamation point (!) indicates the absence of interference. ${ }^{\text {? }}$

\footnotetext{
${ }^{7}$ We suggest that a laboratory study of the sharpness of lines under varying conditions may be of great assistance in interpreting solar and stellar phenomena, when the sharpness of lines in celestial sources shall have been investigated.
} $50239^{\circ}-16-6$ 
TABLE 1

Wave Lengths and Characters of Iron Lines (3233-6750)

\begin{tabular}{|c|c|c|c|c|c|c|c|c|c|c|}
\hline Wave length & I & p. e. & & Order & Meas. & & & Drder $\mathrm{F}$ & Exam. & \\
\hline $\begin{array}{r}3233.056 \\
36.227 \\
39.440 \\
44.189 \\
54.367\end{array}$ & $\begin{array}{l}5 \\
5 \\
8 \\
8 \\
4\end{array}$ & $\begin{array}{l}\mathbf{A} \\
\mathbf{A} \\
\mathbf{B} \\
\mathbf{B} \\
\mathbf{A}\end{array}$ & $\begin{array}{l}23 \\
23 \\
23 \\
23 \\
23\end{array}$ & $\begin{array}{l}31 \\
31 \\
31\end{array}$ & 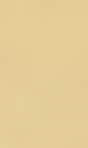 & $\cdot$ & $\begin{array}{l}46 \\
46 \\
18^{*} \\
18^{*} \\
46\end{array}$ & $\begin{array}{l}31^{*} \\
31^{*}\end{array}$ & $\begin{array}{l}46 ! \\
46 !\end{array}$ & \\
\hline $\begin{array}{l}57.598 \\
65.622 \\
71.005 \\
80.264 \\
84.593\end{array}$ & $\begin{array}{l}4 \\
6 \\
6 \mathrm{~b} \\
5 \\
4\end{array}$ & $\begin{array}{l}\mathbf{B} \\
\mathbf{A} \\
\mathbf{A} \\
\mathbf{A} \\
\mathbf{A}\end{array}$ & $\begin{array}{l}23 \\
23 \\
23 \\
23 \\
23\end{array}$ & $\begin{array}{l}31 \\
31 \\
31 \\
30 \\
30\end{array}$ & & & $\begin{array}{l}46 \\
46 ! \\
46 ! \\
46 \\
46\end{array}$ & & & \\
\hline $\begin{array}{r}86.760 \\
90.992 \\
3298.136 \\
3305.977 \\
06.358\end{array}$ & $\begin{array}{l}8 \\
4 \\
5 \\
8 \\
8\end{array}$ & $\begin{array}{l}\mathbf{A} \\
\mathbf{B} \\
\mathbf{A} \\
\mathbf{A} \\
\mathbf{A}\end{array}$ & $\begin{array}{l}23 \\
23 \\
23 \\
23 \\
23\end{array}$ & $\begin{array}{l}30 \\
30 \\
30 \\
30\end{array}$ & $45^{*}$ & & $\begin{array}{l}12^{*} \\
45 !\end{array}$ & $18^{*}$ & $30 !$ & \\
\hline $\begin{array}{l}14.746 \\
23.741 \\
28.870 \\
37.670 \\
47.930\end{array}$ & $\begin{array}{l}6 \\
4 \\
4 \\
4 \\
4\end{array}$ & $\begin{array}{l}\mathbf{A} \\
\mathbf{A} \\
\mathbf{A} \\
\mathbf{A} \\
\mathbf{A}\end{array}$ & $\begin{array}{l}23 \\
23 \\
23 \\
22 \\
22\end{array}$ & $\begin{array}{l}30 \\
30 \\
30 \\
30\end{array}$ & & & $\begin{array}{l}45 \\
45 \\
45 \\
\\
30\end{array}$ & & & \\
\hline $\begin{array}{l}55.232 \\
70.787 \\
79.024 \\
80.115 \\
83.984\end{array}$ & $\begin{array}{l}4 \\
6 \\
4 \\
5 \\
5\end{array}$ & $\begin{array}{l}\mathbf{A} \\
\mathbf{A} \\
\mathbf{B} \\
\mathbf{A} \\
\mathbf{A}\end{array}$ & $\begin{array}{l}22 \\
22 \\
22 \\
22 \\
22\end{array}$ & $\begin{array}{l}30 \\
29 \\
29 \\
29 \\
29\end{array}$ & $\begin{array}{l}45 \\
44 \\
44 \\
44 \\
44\end{array}$ & & & & & \\
\hline $\begin{array}{r}92.658 \\
94.588 \\
96.981 \\
3399.338 \\
3401.523\end{array}$ & $\begin{array}{l}5 \\
4 \\
3 \\
6 \\
4\end{array}$ & $\begin{array}{l}\mathbf{A} \\
\mathbf{A} \\
\mathbf{A} \\
\mathbf{A} \\
\mathbf{A}\end{array}$ & $\begin{array}{l}22 \\
22 \\
22 \\
22 \\
22\end{array}$ & $\begin{array}{l}29 \\
29 \\
29 \\
29 \\
29\end{array}$ & $\begin{array}{l}44 \\
44 \\
44 \\
44\end{array}$ & & 44 & 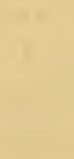 & & \\
\hline $\begin{array}{l}02.261 \\
07.464 \\
13.136 \\
15.537 \\
17.844\end{array}$ & $\begin{array}{l}4 \\
71 \mathrm{~d} \\
7 \\
4 \\
6\end{array}$ & $\begin{array}{l}\mathbf{A} \\
\mathbf{A} \\
\mathbf{A} \\
\mathbf{A} \\
\mathbf{A}\end{array}$ & $\begin{array}{l}22 \\
22 \\
22 \\
22 \\
22\end{array}$ & $\begin{array}{l}29 \\
29 \\
29 \\
29\end{array}$ & $\begin{array}{l}44 \\
44 \\
44 \\
44\end{array}$ & & $18^{*}$ & $29 !$ & & \\
\hline $\begin{array}{l}18.512 \\
24.289 \\
27.122 \\
28.197 \\
31.822\end{array}$ & $\begin{array}{l}5 \\
6 \\
6 \\
6 \\
2 \mathrm{~b}\end{array}$ & $\begin{array}{l}\mathbf{A} \\
\mathbf{A} \\
\mathbf{B} \\
\mathbf{A} \\
\mathbf{C}\end{array}$ & $\begin{array}{l}22 \\
22 \\
22 \\
22 \\
22\end{array}$ & $\begin{array}{l}29 \\
\\
29 \\
29\end{array}$ & 44 & & $\begin{array}{l}29 \\
29 * \\
44 !\end{array}$ & $\begin{array}{l}44 ! \\
44 !\end{array}$ & & \\
\hline $\begin{array}{l}42.366 \\
45.153 \\
47.282 \\
50.332 \\
58.306\end{array}$ & $\begin{array}{l}4 \\
4 \\
6 \\
6 \\
3\end{array}$ & $\begin{array}{l}\text { B } \\
\text { A } \\
\text { A } \\
\text { A } \\
\text { A }\end{array}$ & $\begin{array}{l}22 \\
22 \\
22 \\
22 \\
22\end{array}$ & $\begin{array}{l}29 \\
29 \\
29 \\
29 \\
29\end{array}$ & $\begin{array}{l}44^{*} \\
43 \\
43 \\
43 \\
43\end{array}$ & & & & & \\
\hline $\begin{array}{l}59.916 \\
76.707 \\
85.343 \\
89.673 \\
95.292\end{array}$ & $\begin{array}{l}4 \\
5 r \\
6 \\
4 \\
4 \nabla\end{array}$ & $\begin{array}{l}\mathbf{A} \\
\mathbf{A} \\
\mathbf{A} \\
\mathbf{A} \\
\mathbf{A}\end{array}$ & $\begin{array}{l}22 \\
22 \\
21 \\
21 \\
21\end{array}$ & $\begin{array}{l}29 \\
29 \\
29 \\
29\end{array}$ & $\begin{array}{l}43 \\
43 \\
43\end{array}$ & & $\begin{array}{l}43^{*} \\
11^{*}\end{array}$ & $17^{*}$ & $29 *$ & $43^{*}$ \\
\hline
\end{tabular}


TABLE 1-Continued

Wave Lengths and Characters of Iron Lines (3233-6750)-Continued

\begin{tabular}{|c|c|c|c|c|c|c|c|c|c|c|}
\hline Wave length & I & p. e. & & Order & I Mea & & & Order & Exam. & \\
\hline $\begin{array}{r}97.111 \\
3497.847 \\
3506.501 \\
13.822 \\
21.266\end{array}$ & $\begin{array}{l}4 \\
5 r \\
5 \\
5 \\
5 r\end{array}$ & $\begin{array}{l}\text { B } \\
\text { B } \\
\text { A } \\
\text { A } \\
\text { B }\end{array}$ & $\begin{array}{l}21 \\
21 \\
21 \\
21 \\
21\end{array}$ & 29 & 43 & & $\begin{array}{l}29 \\
11^{*} \\
11^{*} \\
11^{*}\end{array}$ & $\begin{array}{l}43^{*} \\
17^{*} \\
17^{*} \\
17^{*}\end{array}$ & $\begin{array}{l}29 * \\
28 ! \\
28 !\end{array}$ & $43^{*}$ \\
\hline $\begin{array}{l}27.796 \\
29.819 \\
36.558 \\
41.089 \\
42.080\end{array}$ & $\begin{array}{l}4 \\
4 \\
6 \\
6 \mathrm{~h} \\
6\end{array}$ & $\begin{array}{l}\text { B } \\
\text { B } \\
\text { A } \\
\text { A } \\
\text { A }\end{array}$ & $\begin{array}{l}21 \\
21 \\
21 \\
21 \\
21\end{array}$ & $\begin{array}{l}28 \\
28\end{array}$ & & & $\begin{array}{l}42^{*} \\
42^{*} \\
11^{*} \\
11^{*} \\
11^{*}\end{array}$ & $\begin{array}{l}57 ! \\
57 ! \\
28^{*} \\
17^{*} \\
17^{*}\end{array}$ & $\begin{array}{l}42 ! \\
28 ! \\
28 !\end{array}$ & \\
\hline $\begin{array}{l}45.642 \\
54.928 \\
56.878 \\
58.519 \\
60.703\end{array}$ & $\begin{array}{l}5 \\
8 \mathrm{~h} \\
6 \mathrm{~h} \\
5 \mathrm{r} \\
2\end{array}$ & $\begin{array}{l}\mathbf{A} \\
\mathbf{B} \\
\mathbf{A} \\
\mathbf{C} \\
\mathbf{A}\end{array}$ & $\begin{array}{l}21 \\
21 \\
21 \\
21 \\
21\end{array}$ & 28 & 42 & $56 *$ & $\begin{array}{l}42 \\
11^{*} \\
11^{*} \\
11^{*}\end{array}$ & $\begin{array}{l}17^{*} \\
17^{*} \\
17^{*}\end{array}$ & $\begin{array}{l}28 ! \\
28 ! \\
28 *\end{array}$ & $42^{*}$ \\
\hline $\begin{array}{l}71.999 \\
76.762 \\
82.203 \\
86.117 \\
89.108\end{array}$ & $\begin{array}{l}7 \mathrm{~h} \\
4 \\
4 \\
5 \\
4\end{array}$ & $\begin{array}{l}\mathbf{B} \\
\mathbf{A} \\
\mathbf{A} \\
\mathbf{A} \\
\mathbf{A}\end{array}$ & $\begin{array}{l}21 \\
21 \\
21 \\
21 \\
21\end{array}$ & $\begin{array}{l}28 \\
28 \\
28 \\
28 \\
28\end{array}$ & $\begin{array}{l}42 \\
42 \\
42 \\
42\end{array}$ & $\begin{array}{l}56 \\
56 \\
56\end{array}$ & $56 !$ & & & 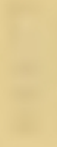 \\
\hline $\begin{array}{r}3594.632 \\
3603.207 \\
06.682 \\
10.159 \\
12.084\end{array}$ & $\begin{array}{l}5 \\
5 \\
5 \\
5 \mathrm{~h} \\
4\end{array}$ & $\begin{array}{l}\mathbf{A} \\
\mathbf{A} \\
\mathbf{A} \\
\mathbf{B} \\
\mathbf{B}\end{array}$ & $\begin{array}{l}21 \\
21 \\
21 \\
21 \\
21\end{array}$ & $\begin{array}{l}28 \\
28 \\
\\
28\end{array}$ & 42 & 56 & $\begin{array}{l}28^{*} \\
42^{*} \\
11^{*} \\
42^{*}\end{array}$ & $\begin{array}{l}42^{*} \\
55 ! \\
17^{*} \\
55 !\end{array}$ & $\begin{array}{l}56 ! \\
28 !\end{array}$ & 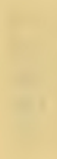 \\
\hline $\begin{array}{l}17.789 \\
21.464 \\
22.007 \\
23.188 \\
25.150\end{array}$ & $\begin{array}{l}6 \\
6 \\
6 \\
5 \\
6\end{array}$ & $\begin{array}{l}\mathbf{A} \\
\mathbf{A} \\
\mathbf{B} \\
\mathbf{A} \\
\mathbf{B}\end{array}$ & $\begin{array}{l}21 \\
21 \\
21 \\
21 \\
21\end{array}$ & $\begin{array}{l}28 \\
28 \\
28 \\
28 \\
28\end{array}$ & $\begin{array}{l}42 \\
41 \\
41 \\
41\end{array}$ & 55 & $\begin{array}{l}55 ! \\
55 ! \\
55^{*} \\
41\end{array}$ & $55 !$ & & \\
\hline $\begin{array}{l}32.044 \\
38.299 \\
40.392 \\
45.825 \\
51.471\end{array}$ & $\begin{array}{l}6 \\
6 \\
6 \\
4 \\
6\end{array}$ & $\begin{array}{l}\mathbf{A} \\
\mathbf{B} \\
\mathbf{A} \\
\mathbf{A} \\
\mathbf{A}\end{array}$ & $\begin{array}{l}21 \\
21 \\
21 \\
21 \\
21\end{array}$ & $\begin{array}{l}28 \\
28 \\
28 \\
27 \\
27\end{array}$ & $\begin{array}{l}41 \\
41 \\
41 \\
41 \\
41\end{array}$ & 55 & $\begin{array}{l}55 \\
55^{*} \\
55\end{array}$ & & & 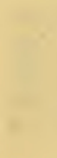 \\
\hline $\begin{array}{l}59.520 \\
69.524 \\
76.313 \\
83.058 \\
84.112\end{array}$ & $\begin{array}{l}5 \\
6 \\
4 \\
4 \\
5\end{array}$ & $\begin{array}{l}\mathbf{A} \\
\mathbf{A} \\
\mathbf{A} \\
\mathbf{B} \\
\mathbf{A}\end{array}$ & $\begin{array}{l}21 \\
20 \\
20 \\
20 \\
20\end{array}$ & $\begin{array}{l}27 \\
27 \\
27 \\
27 \\
27\end{array}$ & $\begin{array}{l}41 \\
41 \\
41 \\
41^{*} \\
41\end{array}$ & $\begin{array}{l}55 \\
54\end{array}$ & $\begin{array}{l}55 \\
54 ! \\
54^{*}\end{array}$ & & & 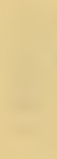 \\
\hline $\begin{array}{r}89.458 \\
3695.055 \\
3701.084 \\
04.465 \\
07.050\end{array}$ & $\begin{array}{l}6 \\
3 \\
6 \\
5 \\
3 \mathrm{~h}\end{array}$ & $\begin{array}{l}\mathbf{B} \\
\mathbf{A} \\
\mathbf{B} \\
\mathbf{A} \\
\mathbf{A}\end{array}$ & $\begin{array}{l}20 \\
20 \\
20 \\
20 \\
20\end{array}$ & $\begin{array}{l}27 \\
27 \\
27 \\
27 \\
27\end{array}$ & $\begin{array}{l}41 \\
40 \\
40^{*}\end{array}$ & $\begin{array}{l}54 \\
54\end{array}$ & $\begin{array}{l}41^{*} \\
41^{*} \\
54 !\end{array}$ & $\begin{array}{l}54 ! \\
54 !\end{array}$ & & \\
\hline $\begin{array}{l}15.915 \\
24.380 \\
31.377 \\
32.401 \\
38.308\end{array}$ & $\begin{array}{l}2 \\
6 \\
1 \\
6 \\
4\end{array}$ & $\begin{array}{l}\mathbf{A} \\
\mathbf{A} \\
\mathbf{A} \\
\mathbf{A} \\
\mathbf{A}\end{array}$ & $\begin{array}{l}20 \\
20 \\
20 \\
20 \\
20\end{array}$ & $\begin{array}{l}27 \\
27 \\
27 \\
27 \\
27\end{array}$ & $\begin{array}{l}40 \\
40 \\
40 \\
40 \\
40\end{array}$ & $\begin{array}{l}54 \\
54 \\
54 \\
\\
54\end{array}$ & $54 !$ & & & \\
\hline
\end{tabular}


TABLE 1-Continued

Wave Lengths and Characters of Iron Lines (3233-6750)-Continued

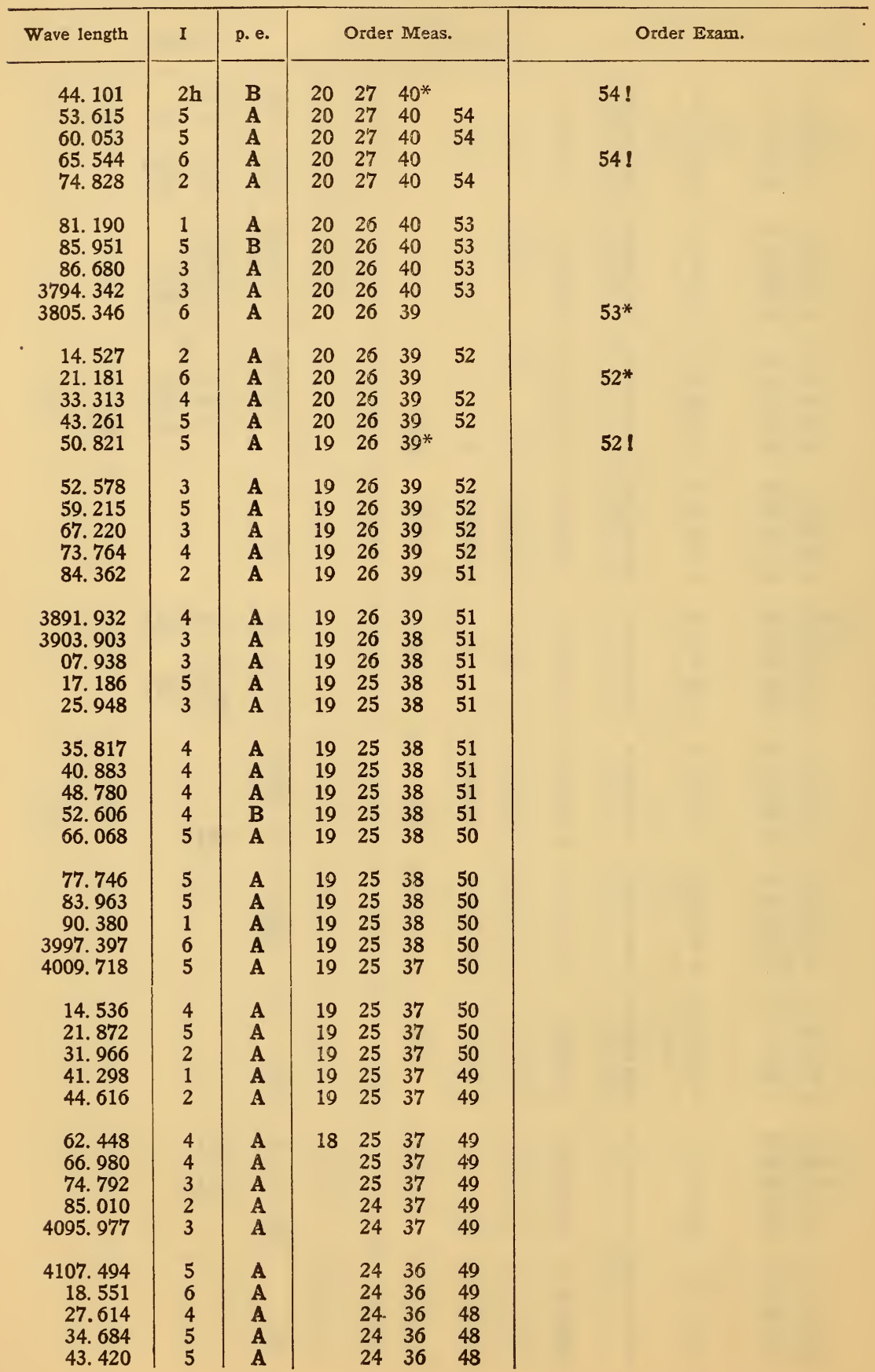


TABLE 1-Continued

Wave Lengths and Characters of Iron Lines (3233-6750)-Continued

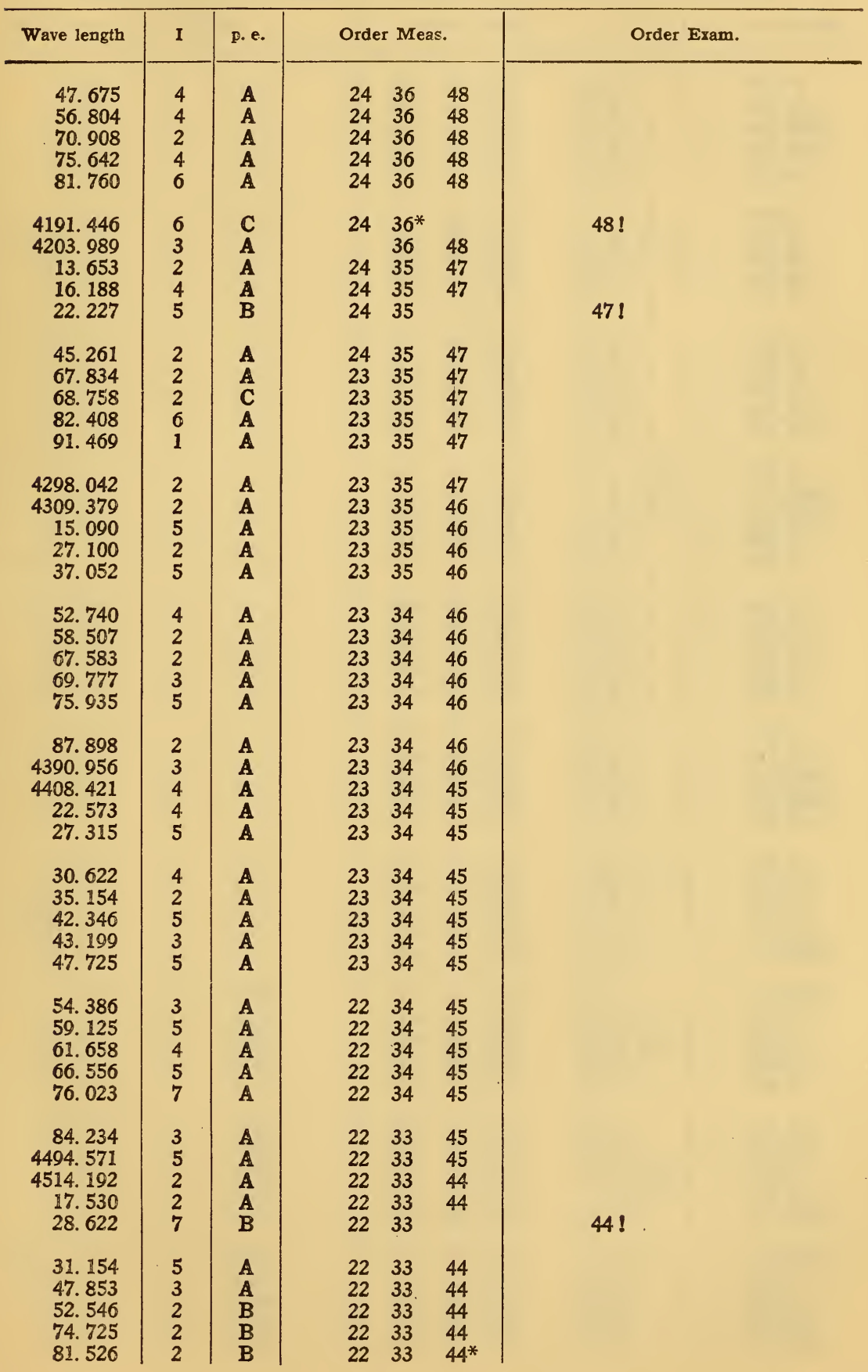


TABLE 1-Continued

Wave Lengths and Characters of Iron Lines (3233-6750)-Continued

\begin{tabular}{|c|c|c|c|c|c|c|}
\hline Wave length & I & p. e. & Order & $\mathrm{r}$ Mea & & Order Exam. \\
\hline $\begin{array}{r}87.135 \\
92.656 \\
4595.364 \\
4602.007 \\
02.946\end{array}$ & $\begin{array}{l}2 \\
4 \\
2 \\
2 \\
4\end{array}$ & $\begin{array}{l}\text { B } \\
\text { A } \\
\text { B } \\
\text { B } \\
\text { A }\end{array}$ & $\begin{array}{l}22 \\
22 \\
22 \\
22 \\
22\end{array}$ & $\begin{array}{l}33 \\
33 \\
33 \\
33 \\
33\end{array}$ & $\begin{array}{l}44 \\
44 \\
44 \\
43 \\
43\end{array}$ & \\
\hline $\begin{array}{l}07.663 \\
13.221 \\
19.299 \\
25.061 \\
30.128\end{array}$ & $\begin{array}{l}4 \\
3 \\
4 \\
4 \\
3\end{array}$ & $\begin{array}{l}\text { B } \\
\mathbf{A} \\
\mathbf{A} \\
\mathbf{A} \\
\mathbf{A}\end{array}$ & $\begin{array}{l}22 \\
22 \\
22 \\
22 \\
22\end{array}$ & $\begin{array}{l}33 \\
33 \\
32 \\
32 \\
32\end{array}$ & $\begin{array}{l}43 \\
43 \\
43 \\
43 \\
43\end{array}$ & \\
\hline $\begin{array}{l}32.917 \\
37.519 \\
47.439 \\
67.461 \\
68.152\end{array}$ & $\begin{array}{l}3 \\
4 \\
4 \\
4 \\
4\end{array}$ & $\begin{array}{l}\mathbf{A} \\
\mathbf{A} \\
\mathbf{A} \\
\mathbf{A} \\
\mathbf{A}\end{array}$ & $\begin{array}{l}22 \\
22 \\
22 \\
21 \\
21\end{array}$ & $\begin{array}{l}32 \\
32 \\
32 \\
32 \\
32\end{array}$ & $\begin{array}{l}43 \\
43 \\
43 \\
43 \\
43\end{array}$ & \\
\hline $\begin{array}{r}78.857 \\
4691.417 \\
4707.289 \\
10.288 \\
33.597\end{array}$ & $\begin{array}{l}5 \\
4 \\
5 \\
3 \\
3\end{array}$ & $\begin{array}{l}\mathbf{A} \\
\mathbf{A} \\
\mathbf{A} \\
\mathbf{A} \\
\mathbf{A}\end{array}$ & $\begin{array}{l}21 \\
21 \\
21 \\
21 \\
21\end{array}$ & $\begin{array}{l}32 \\
32 \\
32 \\
32 \\
32\end{array}$ & $\begin{array}{l}43 \\
43 \\
42 \\
42 \\
42\end{array}$ & \\
\hline $\begin{array}{l}35.849 \\
36.790 \\
41.535 \\
57.585 \\
72.820\end{array}$ & $\begin{array}{l}2 \\
5 \\
3 \\
2 \\
3\end{array}$ & $\begin{array}{l}\text { A } \\
\text { A } \\
\text { A } \\
\text { B } \\
\text { A }\end{array}$ & $\begin{array}{l}21 \\
21 \\
21 \\
21 \\
21\end{array}$ & $\begin{array}{l}32 \\
32 \\
32 \\
32 \\
31\end{array}$ & $\begin{array}{l}42 \\
42 \\
42 \\
42 \\
42\end{array}$ & \\
\hline $\begin{array}{r}86.812 \\
88.763 \\
4789.657 \\
4802.887 \\
39.551\end{array}$ & $\begin{array}{l}3 \\
2 \\
3 \\
2 \\
2\end{array}$ & $\begin{array}{l}\text { A } \\
\text { A } \\
\text { A } \\
\text { B } \\
\text { B }\end{array}$ & $\begin{array}{l}21 \\
21 \\
21 \\
21 \\
21\end{array}$ & $\begin{array}{l}31 \\
31 \\
31 \\
31 \\
31\end{array}$ & $\begin{array}{l}42 \\
42 \\
42 \\
42 \\
41\end{array}$ & \\
\hline $\begin{array}{r}59.756 \\
71.333 \\
72.154 \\
78.227 \\
4890.771\end{array}$ & $\begin{array}{l}5 \\
8 \\
6 \\
5 \\
7\end{array}$ & $\begin{array}{l}\text { B } \\
\text { A } \\
\text { A } \\
\text { A } \\
\text { B }\end{array}$ & $\begin{array}{l}21 \\
21 \\
21 \\
20 \\
20\end{array}$ & $\begin{array}{l}31 \\
31 \\
31 \\
31 \\
31\end{array}$ & $\begin{array}{l}41 \\
41 \\
41 \\
41 \\
41\end{array}$ & \\
\hline $\begin{array}{r}4903.326 \\
19.009 \\
24.777 \\
38.828 \\
39.691\end{array}$ & $\begin{array}{l}5 \\
8 \\
3 \\
5 \\
3\end{array}$ & $\begin{array}{l}\mathbf{A} \\
\mathbf{A} \\
\mathbf{A} \\
\mathbf{A} \\
\mathbf{A}\end{array}$ & $\begin{array}{l}20 \\
20 \\
20 \\
20 \\
20\end{array}$ & $\begin{array}{l}31 \\
31 \\
30 \\
30 \\
30\end{array}$ & $\begin{array}{l}41 \\
41 \\
41 \\
41 \\
41\end{array}$ & $\begin{array}{l}61 ! \\
61 ! \\
61 ! \\
61 ! \\
61\end{array}$ \\
\hline $\begin{array}{r}46.403 \\
66.106 \\
73.116 \\
78.617 \\
4994.133\end{array}$ & $\begin{array}{l}2 \\
5 \\
2 \\
2 \\
3\end{array}$ & $\begin{array}{l}\mathbf{A} \\
\mathbf{A} \\
\mathbf{A} \\
\mathbf{A} \\
\mathbf{A}\end{array}$ & $\begin{array}{l}20 \\
20 \\
20 \\
20 \\
20\end{array}$ & $\begin{array}{l}30 \\
30 \\
30 \\
30 \\
30\end{array}$ & $\begin{array}{l}40 \\
40 \\
40 \\
40 \\
40\end{array}$ & $\begin{array}{l}61 ! \\
60 ! \\
60 ! \\
60 ! \\
60\end{array}$ \\
\hline $\begin{array}{r}5001.879 \\
12.072 \\
14.958 \\
22.250 \\
28.130\end{array}$ & $\begin{array}{l}5 \\
4 \\
4 \\
4 \\
2\end{array}$ & $\begin{array}{l}\mathrm{A} \\
\mathrm{A} \\
\mathrm{A} \\
\mathrm{A} \\
\mathrm{A}\end{array}$ & $\begin{array}{l}20 \\
20 \\
20 \\
20 \\
20\end{array}$ & $\begin{array}{l}30 \\
30 \\
30 \\
30 \\
30\end{array}$ & $\begin{array}{l}40 \\
40 \\
40 \\
40 \\
40\end{array}$ & $\begin{array}{l}60 ! \\
60 \\
60 \\
60 ! \\
60\end{array}$ \\
\hline
\end{tabular}


TABLE 1-Continued

Wave Lengths and Characters of Iron Lines (3233-6750) - Continued

\begin{tabular}{|c|c|c|c|c|c|c|c|}
\hline \multirow{2}{*}{$\begin{array}{r}\text { Wave length } \\
41.076 \\
41.759 \\
49.827 \\
51.638 \\
68.782\end{array}$} & \multirow{2}{*}{$\begin{array}{l}\text { I } \\
3 \\
4 \\
5 \\
4 \\
4\end{array}$} & \multirow{2}{*}{$\begin{array}{l}\text { p. e. } \\
\\
\text { A } \\
\text { A } \\
\text { A } \\
\text { A } \\
\text { A }\end{array}$} & \multicolumn{3}{|c|}{ Order Meas. } & \multicolumn{2}{|c|}{ Order Exam. } \\
\hline & & & $\begin{array}{l}20 \\
20 \\
20 \\
20 \\
20\end{array}$ & $\begin{array}{l}30 \\
30 \\
30 \\
30 \\
30\end{array}$ & $\begin{array}{l}40 \\
40 \\
40 \\
40 \\
39\end{array}$ & $\begin{array}{l}59 \\
59 \\
59 \\
59 \\
59 !\end{array}$ & \\
\hline $\begin{array}{r}83.343 \\
5098.709 \\
5110.414 \\
23.724 \\
27.364\end{array}$ & $\begin{array}{l}4 \\
4 \\
4 \\
4 \\
3\end{array}$ & $\begin{array}{l}\mathbf{A} \\
\mathbf{C} \\
\mathbf{A} \\
\mathbf{A} \\
\mathbf{A}\end{array}$ & $\begin{array}{l}20 \\
20 \\
20 \\
20\end{array}$ & $\begin{array}{l}30 \\
29 \\
29 \\
29 \\
29\end{array}$ & $\begin{array}{l}39 \\
39 \\
39 \\
39 \\
39\end{array}$ & $\begin{array}{l}59 \\
20 \\
59 \\
59 \\
58\end{array}$ & 59 \\
\hline $\begin{array}{l}31.473 \\
41.745 \\
50.844 \\
51.914 \\
66.285\end{array}$ & $\begin{array}{l}2 \\
2 \\
4 \\
3 \\
3\end{array}$ & $\begin{array}{l}\mathbf{A} \\
\mathbf{A} \\
\mathbf{A} \\
\mathbf{A} \\
\mathbf{A}\end{array}$ & $\begin{array}{l}19 \\
19 \\
19 \\
19 \\
19\end{array}$ & $\begin{array}{l}29 \\
29 \\
29 \\
29 \\
29\end{array}$ & $\begin{array}{l}39 \\
39 \\
39 \\
39 \\
39\end{array}$ & $\begin{array}{l}58 \\
58 \\
58 \\
58 \\
58\end{array}$ & \\
\hline $\begin{array}{r}67.495 \\
71.600 \\
91.471 \\
92.362 \\
5198.716\end{array}$ & $\begin{array}{l}8 \\
7 \\
7 \\
8 \\
4\end{array}$ & $\begin{array}{l}\mathbf{C} \\
\mathbf{A} \\
\mathbf{A} \\
\mathbf{A} \\
\mathbf{A}\end{array}$ & $\begin{array}{l}19 \\
19 \\
19 \\
19\end{array}$ & $\begin{array}{l}29 \\
29 \\
29 \\
29 \\
29\end{array}$ & $\begin{array}{l}39 \\
39 \\
38 \\
38 \\
38\end{array}$ & $\begin{array}{l}19 \\
58 \\
58 ! \\
58\end{array}$ & $\begin{array}{l}58 ! \\
58\end{array}$ \\
\hline $\begin{array}{r}5202.340 \\
08.609 \\
16.278 \\
25.529 \\
32.957\end{array}$ & $\begin{array}{l}5 \\
4 \\
5 \\
2 \\
8\end{array}$ & $\begin{array}{l}\mathbf{A} \\
\mathbf{A} \\
\mathbf{A} \\
\mathbf{A} \\
\mathbf{A}\end{array}$ & $\begin{array}{l}19 \\
19 \\
19 \\
19 \\
19\end{array}$ & $\begin{array}{l}29 \\
29 \\
29 \\
29 \\
29\end{array}$ & $\begin{array}{l}38 \\
38 \\
38 \\
38 \\
38\end{array}$ & $\begin{array}{l}58 \\
58 \\
57 \\
57 \\
57 !\end{array}$ & \\
\hline $\begin{array}{l}35.395 \\
42.496 \\
50.651 \\
63.321 \\
66.572\end{array}$ & $\begin{array}{l}2 \\
3 \\
3 \\
5 \\
8\end{array}$ & $\begin{array}{l}\text { A } \\
\text { A } \\
\text { A } \\
\text { A } \\
\text { A }\end{array}$ & $\begin{array}{l}19 \\
19 \\
19 \\
19 \\
19\end{array}$ & $\begin{array}{l}29 \\
29 \\
29 \\
28 \\
28\end{array}$ & $\begin{array}{l}38 \\
38 \\
38 \\
38 \\
38\end{array}$ & $\begin{array}{l}57 \\
57 \\
57 \\
57^{*} \\
57 !\end{array}$ & \\
\hline $\begin{array}{r}70.360 \\
81.807 \\
5283.636 \\
5302.315 \\
07.364\end{array}$ & $\begin{array}{l}8 \\
5 \\
7 \\
5 \\
2\end{array}$ & $\begin{array}{l}\text { C } \\
\text { A } \\
\text { A } \\
\mathbf{A} \\
\mathbf{B}\end{array}$ & $\begin{array}{l}19 \\
19 \\
19 \\
19\end{array}$ & $\begin{array}{l}28 \\
28 \\
28 \\
28 \\
28\end{array}$ & $\begin{array}{l}38 \\
38 \\
38 \\
38 \\
38\end{array}$ & $\begin{array}{l}19 \\
57^{*} \\
57^{*} \\
57 ! \\
57\end{array}$ & $57 !$ \\
\hline $\begin{array}{l}22.047 \\
24.195 \\
28.538 \\
32.902 \\
41.027\end{array}$ & $\begin{array}{l}2 \\
6 \\
4 \\
2 \\
5\end{array}$ & $\begin{array}{l}\text { C } \\
\text { A } \\
\text { B } \\
\text { A } \\
\text { A }\end{array}$ & $\begin{array}{l}19 \\
19 \\
19 \\
19\end{array}$ & $\begin{array}{l}28 \\
28 \\
28 \\
28 \\
28\end{array}$ & $\begin{array}{l}38 \\
38 \\
38 \\
38 \\
37\end{array}$ & $\begin{array}{l}56 ! \\
56 ! \\
19 \\
56 \\
56\end{array}$ & $56 *$ \\
\hline $\begin{array}{l}53.385 \\
65.403 \\
71.497 \\
79.579 \\
93.183\end{array}$ & $\begin{array}{l}2 \\
2 \\
7 \\
2 \\
4\end{array}$ & $\begin{array}{l}\mathbf{A} \\
\mathbf{A} \\
\mathbf{C} \\
\mathbf{A} \\
\mathbf{A}\end{array}$ & $\begin{array}{l}19 \\
19 \\
19 \\
19\end{array}$ & $\begin{array}{l}28 \\
28 \\
28 \\
28 \\
28\end{array}$ & $\begin{array}{l}37 \\
37 \\
37 \\
37\end{array}$ & $\begin{array}{l}56 ! \\
56 \\
19 \\
56 !\end{array}$ & $\begin{array}{l}37 ! \\
56\end{array}$ \\
\hline $\begin{array}{r}5397.132 \\
5405.781 \\
29.701 \\
34.528 \\
46.920\end{array}$ & $\begin{array}{l}6 \\
6 \\
6 \\
6 \\
6\end{array}$ & $\begin{array}{l}\mathbf{A} \\
\mathbf{A} \\
\mathbf{A} \\
\mathbf{A} \\
\mathbf{A}\end{array}$ & $\begin{array}{l}19 \\
19 \\
18 \\
18 \\
18\end{array}$ & $\begin{array}{l}28 \\
28 \\
28 \\
28 \\
28\end{array}$ & $\begin{array}{l}37 \\
37 \\
37 \\
37 \\
37\end{array}$ & $\begin{array}{l}56 ! \\
55 ! \\
55 ! \\
55 \\
55 !\end{array}$ & \\
\hline
\end{tabular}


TABLE 1-Continued

Wave Lengths and Characters of Iron Lines (3233-6750)-Continued

\begin{tabular}{|c|c|c|c|c|c|c|c|c|}
\hline Wave length & I & p. e. & Orde & $\mathrm{Mea}$ & & & Drder & Exam. \\
\hline $\begin{array}{r}55.614 \\
73.913 \\
5497.521 \\
5501.468 \\
06.783\end{array}$ & $\begin{array}{l}6 \\
3 \\
4 \\
4 \\
4\end{array}$ & $\begin{array}{l}\mathbf{A} \\
\mathbf{A} \\
\mathbf{A} \\
\mathbf{A} \\
\mathbf{A}\end{array}$ & $\begin{array}{l}18 \\
18 \\
18 \\
18 \\
18\end{array}$ & $\begin{array}{l}27 \\
27 \\
27 \\
27 \\
27\end{array}$ & $\begin{array}{l}37 \\
37 \\
37 \\
37 \\
37\end{array}$ & $\begin{array}{l}55 ! \\
55 ! \\
55 \\
54 \\
54\end{array}$ & & \\
\hline $\begin{array}{l}25.557 \\
35.423 \\
43.184 \\
43.953 \\
63.611\end{array}$ & $\begin{array}{l}2 \\
2 \\
2 \\
2 \\
3\end{array}$ & $\begin{array}{l}\text { B } \\
\text { B } \\
\text { C } \\
\text { B } \\
\text { B }\end{array}$ & $\begin{array}{l}18 \\
18 \\
18 \\
18 \\
18\end{array}$ & $\begin{array}{l}27 \\
27 \\
27 \\
27 \\
27\end{array}$ & $\begin{array}{l}37 \\
37 \\
37 \\
37 \\
37\end{array}$ & $\begin{array}{l}54 ! \\
54 ! \\
54 ! \\
54 ! \\
54 !\end{array}$ & & \\
\hline $\begin{array}{r}69.633 \\
72.859 \\
76.106 \\
5586.772 \\
5615.661\end{array}$ & $\begin{array}{l}5 \\
5 \\
4 \\
6 \\
6\end{array}$ & $\begin{array}{l}\mathbf{A} \\
\mathbf{A} \\
\mathbf{A} \\
\mathbf{A} \\
\mathbf{B}\end{array}$ & $\begin{array}{l}18 \\
18 \\
18 \\
18 \\
18\end{array}$ & $\begin{array}{l}27 \\
27 \\
27 \\
27 \\
27\end{array}$ & $\begin{array}{l}37 \\
37 \\
37 \\
36 \\
36\end{array}$ & $\begin{array}{l}54^{*} \\
54^{*} \\
54^{*} \\
54^{*} \\
53 !\end{array}$ & & \\
\hline $\begin{array}{l}\text { 18. } 646 \\
24.559 \\
38.276 \\
41.458 \\
55.505\end{array}$ & $\begin{array}{l}1 \\
5 \\
3 \\
2 \\
2\end{array}$ & $\begin{array}{l}\text { B } \\
\text { B } \\
\text { A } \\
\text { C } \\
\text { C }\end{array}$ & $\begin{array}{l}18 \\
18 \\
18 \\
18 \\
18\end{array}$ & $\begin{array}{l}27 \\
27 \\
27\end{array}$ & $\begin{array}{l}36 \\
36 \\
35\end{array}$ & $\begin{array}{l}53^{*} \\
53 ! \\
27 \\
27^{*}\end{array}$ & $\begin{array}{l}35^{*} \\
35 !\end{array}$ & $53 !$ \\
\hline $\begin{array}{r}58.835 \\
62.534 \\
5693.638 \\
5701.553 \\
09.395\end{array}$ & $\begin{array}{l}4 \\
3 \\
2 \\
3 \\
3\end{array}$ & $\begin{array}{l}\mathbf{B} \\
\mathbf{A} \\
\mathbf{B} \\
\mathbf{A} \\
\mathbf{A}\end{array}$ & $\begin{array}{l}18 \\
18 \\
18 \\
18 \\
17\end{array}$ & $\begin{array}{l}26 \\
26 \\
26 \\
26 \\
26\end{array}$ & $\begin{array}{l}35 \\
35 \\
35 \\
35 \\
35\end{array}$ & $\begin{array}{l}53^{*} \\
53 ! \\
53^{*} \\
53^{*}\end{array}$ & & \\
\hline $\begin{array}{l}17.853 \\
31.774 \\
41.865 \\
53.142 \\
63.013\end{array}$ & $\begin{array}{l}3 \\
3 \\
2 \\
3 \\
4\end{array}$ & $\begin{array}{l}\mathbf{B} \\
\mathbf{B} \\
\mathbf{C} \\
\mathbf{A} \\
\mathbf{A}\end{array}$ & $\begin{array}{l}17 \\
17 \\
17 \\
17 \\
17\end{array}$ & $\begin{array}{l}26 \\
26 \\
26 \\
26\end{array}$ & $\begin{array}{l}35 \\
35 \\
\\
35 \\
35\end{array}$ & $\begin{array}{l}52 ! \\
26 \\
52^{*} \\
52^{*}\end{array}$ & $35 !$ & \\
\hline $\begin{array}{r}75.099 \\
91.045 \\
93.936 \\
5798.198 \\
5862.348\end{array}$ & $\begin{array}{l}3 \\
2 \\
2 \\
2 \\
4 \mathrm{~b}\end{array}$ & $\begin{array}{l}\text { B } \\
\text { B } \\
\text { C } \\
\text { C } \\
\text { C }\end{array}$ & $\begin{array}{l}17 \\
17 \\
17 \\
17 \\
17\end{array}$ & $\begin{array}{l}26 \\
26 \\
26 \\
26\end{array}$ & $\begin{array}{l}35 \\
34 \\
34\end{array}$ & $\begin{array}{l}52 * \\
34 ! \\
26 !\end{array}$ & & \\
\hline $\begin{array}{r}5883.842 \\
5905.682 \\
16.252 \\
34.681 \\
75.352\end{array}$ & $\begin{array}{l}4 \\
2 \\
3 \\
4 \\
2\end{array}$ & $\begin{array}{l}\text { C } \\
\text { C } \\
\text { B } \\
\text { B } \\
\text { A }\end{array}$ & $\begin{array}{l}17 \\
17 \\
17 \\
17 \\
17\end{array}$ & $\begin{array}{l}25 \\
25 \\
25\end{array}$ & $\begin{array}{l}34 \\
34 \\
33\end{array}$ & $\begin{array}{l}26^{*} \\
25^{*} \\
51 ! \\
50 !\end{array}$ & $\begin{array}{l}34^{*} \\
34^{*}\end{array}$ & $51 !$ \\
\hline $\begin{array}{r}5976.804 \\
6003.037 \\
08.582 \\
27.059 \\
42.092\end{array}$ & $\begin{array}{l}2 \\
3 \\
3 \\
2 \\
2\end{array}$ & $\begin{array}{l}\mathbf{B} \\
\mathbf{B} \\
\mathbf{B} \\
\mathbf{A} \\
\mathbf{A}\end{array}$ & $\begin{array}{l}17 \\
17 \\
17 \\
17 \\
17\end{array}$ & $\begin{array}{l}25 \\
25 \\
25 \\
25 \\
25\end{array}$ & $\begin{array}{l}33 \\
33 \\
33 \\
33 \\
33\end{array}$ & & & \\
\hline $\begin{array}{r}55.990 \\
65.492 \\
78.484 \\
6089.570 \\
6102.185\end{array}$ & $\begin{array}{l}3 \mathrm{~h} \\
4 \\
3 \\
1 \\
3\end{array}$ & $\begin{array}{l}\text { B } \\
\text { A } \\
\text { B } \\
\text { B } \\
\text { C }\end{array}$ & $\begin{array}{l}17 \\
16 \\
16 \\
16 \\
16\end{array}$ & $\begin{array}{l}25 \\
25 \\
24^{*}\end{array}$ & 33 & $\begin{array}{l}25^{*} \\
49 \\
25^{*} \\
\\
33 !\end{array}$ & $33 !$ & \\
\hline
\end{tabular}


TABLE 1-Continued

Wave Lengths and Characters of Iron Lines (3233-6750)-Continued

\begin{tabular}{|c|c|c|c|c|c|c|c|}
\hline \multirow{2}{*}{$\begin{array}{c}\text { Wave length } \\
27.914 \\
36.625 \\
37.700 \\
41.758 \\
51.632\end{array}$} & \multirow{2}{*}{$\begin{array}{l}\text { I } \\
2 \\
4 \\
4\end{array}$} & \multirow{2}{*}{$\begin{array}{l}\text { p.e. } \\
\text { A } \\
\text { A } \\
\text { A } \\
\text { B } \\
\text { C }\end{array}$} & \multicolumn{3}{|c|}{ Order Meas. } & \multicolumn{2}{|r|}{ Order Exam. } \\
\hline & & & $\begin{array}{l}16 \\
16 \\
16 \\
16\end{array}$ & $\begin{array}{l}24 \\
24 \\
24 \\
24 \\
24\end{array}$ & $\begin{array}{l}33 \\
33 \\
33 \\
33 \\
32\end{array}$ & $\begin{array}{l}49 \\
49 \\
16\end{array}$ & \\
\hline 57. 736 & 2 & B & 16 & 24 & 32 & & \\
\hline 65.370 & 2 & B & 16 & & 32 & & \\
\hline 70.495 & $2 b$ & C & 16 & & & $24^{*}$ & $32 !$ \\
\hline 73. 344 & 2 & B & 16 & 24 & 32 & & \\
\hline 6191.569 & 5 & $\mathbf{A}$ & 16 & 24 & 32 & 49 & \\
\hline 6200.323 & 2 & A & 16 & 24 & 32 & & \\
\hline 13.440 & 3 & $\mathbf{A}$ & 16 & 24 & 32 & 48 & \\
\hline 15. 152 & 2 & B & 16 & 24 & 32 & & \\
\hline 19. 290 & 3 & $\overline{\mathbf{A}}$ & 16 & 24 & 32 & 48 & \\
\hline 30.734 & 5 & $\mathbf{A}$ & 16 & 24 & 32 & 48 & \\
\hline 32.667 & 2 & $\mathbf{A}$ & 16 & 24 & 32 & & \\
\hline 40.660 & 2 & B & 16 & 24 & 32 & & \\
\hline 46.340 & 4 & $\mathbf{A}$ & 16 & 24 & 32 & $48^{*}$ & \\
\hline 52.567 & 4 & $\mathbf{A}$ & 16 & 24 & 32 & 48 & \\
\hline 54.266 & 3 & A & 16 & 24 & 32 & 48 & \\
\hline 56.372 & 3 & $\mathbf{A}$ & 16 & 24 & 32 & 48 & \\
\hline 65.143 & 3 & A & 16 & 24 & 32 & 48 & \\
\hline 70. 236 & 2 & C & & 24 & 32 & 16 & \\
\hline 80.625 & 2 & $\mathbf{A}$ & 16 & 24 & 32 & & \\
\hline 6297.802 & 3 & B & 16 & 24 & 32 & 48 & \\
\hline 6301.524 & 5 & B & 16 & 24 & 32 & 48 & \\
\hline 02.515 & 3 & B & 16 & 24 & 32 & 48 & \\
\hline 18. 028 & 4 & $\overline{\mathbf{A}}$ & 16 & 24 & 32 & 47 & \\
\hline 22. 697 & 3 & A & 16 & 24 & 32 & & \\
\hline 35. 342 & 4 & A & 16 & 24 & 32 & 47 & \\
\hline 36.846 & 4 & A & 16 & 24 & 32 & 47 & \\
\hline 44. 159 & 2 & B & 16 & 24 & 32 & & \\
\hline 55.042 & 3 & $\mathbf{B}$ & 16 & 24 & 31 & & \\
\hline 80.753 & 3 & B & 16 & 23 & 31 & & \\
\hline 6393.612 & 5 & $\overline{\mathbf{A}}$ & 16 & 23 & 31 & 47 & \\
\hline 6400.027 & 5 & A & 16 & 23 & 31 & $47^{*}$ & \\
\hline 6408.044 & 4 & B & & 23 & 31 & 47 & \\
\hline 11.674 & 5 & $\overline{\mathbf{A}}$ & 16 & 23 & 31 & 47 & \\
\hline 21.360 & 4 & $\mathbf{A}$ & 16 & 23 & 31 & 47 & \\
\hline 30.857 & 5 & A & 16 & 23 & 31 & 47 & \\
\hline 62.737 & 4 & A & 15 & 23 & 31 & & \\
\hline 75.639 & 3 & $\mathrm{C}$ & 15 & 23 & 31 & & \\
\hline 81.882 & 3 & $\mathbf{A}$ & 15 & 23 & 31 & & \\
\hline 6494.994 & 5 & A & 15 & 23 & 31 & 46 & \\
\hline 6518.382 & 3 & B & 15 & 23 & 31 & & \\
\hline 46.253 & 5 & A & 15 & 23 & 31 & 46 & \\
\hline
\end{tabular}


TABLE 1- Continued

Wave Lengths and Characters of Iron Lines (3233-6750)-Continued

\begin{tabular}{|c|c|c|c|c|c|c|}
\hline Wave length & I & p. e. & Orde & $\mathrm{Me}$ & & Order Exam. \\
\hline $\begin{array}{r}75.029 \\
92.926 \\
6593.884 \\
6609.125 \\
63.454\end{array}$ & $\begin{array}{l}3 \\
5 \\
3 \\
4 \\
4\end{array}$ & $\begin{array}{l}\mathbf{A} \\
\mathbf{A} \\
\mathbf{C} \\
\mathbf{A} \\
\mathbf{A}\end{array}$ & $\begin{array}{l}15 \\
15 \\
15 \\
15 \\
15\end{array}$ & $\begin{array}{l}23 \\
23 \\
23 \\
23 \\
22\end{array}$ & $\begin{array}{l}30 \\
30 \\
30 \\
30 \\
30\end{array}$ & $\begin{array}{l}46 \\
46 \\
45\end{array}$ \\
\hline $\begin{array}{l}6678.000 \\
6750.164\end{array}$ & $\begin{array}{l}5 \\
4\end{array}$ & $\begin{array}{l}\mathbf{A} \\
\mathbf{B}\end{array}$ & $\begin{array}{l}15 \\
15\end{array}$ & $\begin{array}{l}22 \\
22\end{array}$ & $\begin{array}{l}30 \\
30\end{array}$ & $\begin{array}{l}45 \\
44\end{array}$ \\
\hline
\end{tabular}

NOTES TO TABLE 1.-OTHER INTERFERENCE OBSERVATIONS

3271. Buisson and Fabry, ${ }^{1} 0.003$; Pfund, ${ }^{2} 0 . \cos$.

3323. Buisson and Fabry, 0.739; Pfund, 0.738 .

3536 . This wave length may have been affected by a neighboring line.

4678. Buisson and Fabry, 0.855 .

5127. Buisson and Fabry, 0.364 .

5535. Buisson and Fabry, 0.418 .

5934. Buisson and Fabry, 0.683 .

6003 . Buisson and Fabry, 0.039 ; Eversheim, ${ }^{3} 0.039$.

6 I27. The value formerly obtained by one of us, $0.9 \mathrm{I} 9$, was not used in taking the mean on account of the large discrepancy.

6663. Eversheim, 0.449 .

${ }^{1}$ See note 4, p. $248 . \quad 2$ Unpublished. $\quad{ }^{3}$ Eversheim, Annal. der Phys., 4th series, 45, p. 454; 19i4.

\section{AGREEMENT OF STANDARDS}

\section{GENERAL}

The fact that for 15 standards the difference, Bureau of Standards minus International, could be reasonably well represented by a straight line permits a determination of the relative accuracy of the individual I. A. standards. ${ }^{8}$

\section{STANDARDS FOR WHICH B. S.-I. A. IS GREATER THAN 0.001A}

Eighty-one standards were measured. Expressed in $0.001 \mathrm{~A}$, the differences B. S. minus I. A. are as follows: 0,$27 ; \pm \mathrm{I}, 35 ; \pm 2, \mathrm{I} 2$; $\pm 3,5 ; \pm 4,2$. The following table contains all standards for which this difference is over $0.001 \mathrm{~A}$. Differences of $0.002 \mathrm{~A}$ are to be expected occasionally, as the probable error of the standard is sometimes quite large. In Table 2 the first column contains the $B$. S. value; the second, the I. A.; the third, some recent determinations by Eversheim; and the last column contains the p. e. of the standard. 
TABLE 2

Standards Differing by More than 0.001A from I. A. Values

\begin{tabular}{c|c|c|c|c|c|c|c}
\hline Wave lengths & I. A. & E. & p. e. & Wave lengths & I. A. & E. & p. e. \\
\cline { 2 - 7 } 3370.787 & 789 & & 0.6 & 5001.879 & 881 & & 1.4 \\
3485.343 & 345 & & .6 & 5167.495 & 492 & & .8 \\
3556.878 & 881 & & 1.0 & 5266.572 & 569 & & .2 \\
4191.446 & 443 & & 1.0 & 5371.497 & 495 & & 1.2 \\
4592.656 & 658 & & .2 & 6265.143 & 145 & 145 & 1.7 \\
4736.790 & 786 & & .6 & 6430.857 & 859 & 856 & 1.4 \\
4859.756 & 758 & & .6 & 6546.253 & 250 & 250 & 1.2 \\
4878.227 & 225 & & .4 & 6592.926 & 928 & 920 & 1.2 \\
4919.009 & 007 & & .4 & 6678.000 & 004 & 997 & 1.6 \\
4966.106 & 104 & & .4 & & & & \\
\hline
\end{tabular}

\section{NOTES TO TABLE 2}

$355^{6}$ and 4 I 9 I are poor lines which could be measured only with a small order of interference. They had little weight in the final results.

The B. S. value of $5^{1} 67$ is poorly determined, being marked " $\mathrm{C}$ " 'it is not meant as a definitive wave length.

5266 was measured on I $_{5}$ exposures, only 5 of which gave values less than 0.572 .

4736 and 6678 had no weight in the reductions. It is to be noted that Eversheim's most recent determination is more in accord with $B$. S. value than was his value which entered into the mean which is published as I. A.

6546.247 was used by the international committee as one of three observations, the mean of which is the I. A. wave length. This is a typographical error. The correct value is 6546.254 , and the resulting mean is 0.252 , which causes the discrepancy B. S. minus I. A. to vanish in the case of this line.

\section{UNSATISFACTORY STANDARDS}

The line 3677 was measured on all the plates used in this region, yet it was excluded from Table $\mathrm{I}$ on account of the large discrepancies found in the values obtained for it. The following lines are too poor on all of our plates: $3865,3906,4076,4233$. The following standards are lacking in sharpness compared with other lines in their neighborhoods: $35^{1} 3,355^{6}, 3850,4191,5371$.

\section{POLE EFFECT AND INTERFERENCE MEASUREMENTS}

Between 4707 and 5324, inclusive, and between 5569 and 5763 , inclusive, the greater part of the standards have been found by St. John and Babcock ${ }^{10}$ to have longer wave lengths at the pole than in the center of the arc. Now, the standard wave lengths were not observed under normal conditions and the present series was observed under the conditions specified by the international committee. So if, when measured by interference, these lines were displaced by the change in conditions, we should have a 
break in our reduction curve at $4700 \mathrm{~A}$, also at $5350 \mathrm{~A}, 55 \mathrm{roA}$, and $5800 \mathrm{~A}$. There being no standards between $5763 \mathrm{~A}$ and $6027 \mathrm{~A}$, it is impossible to say whether or not a break occurs at $5800 \mathrm{~A}$. It is very evident that no break of over o.oor A occurs at any of the other points, in fact, there is no evidence of any break at all. This leads us to suppose that such interference observations as have been made give consistent results, regardless of the character of the lines in reference to pole shift. It is to be remembered that the interferometer uses a small portion of the center of the arc, where Goos ${ }^{11}$ and St. John and Babcock agree that shifts are not to be feared. Thus it seems probable that the measurement of additional lines on the plates from which the I. A. standards were obtained would lead to the same values of the wave lengths as though the plates had been taken under normal conditions. It follows that an enormous amount of valuable data might be obtained from these plates at the expense of a relatively small amount of labor. It is to be hoped that the spectroscopists who cooperated in the determination of the I. A. standards may be induced to measure a large number of additional lines on the plates which were used in this determination.

\section{COMPARISON WITH GRATING MEASUREMENTS}

\section{GENERAL AGREEMENT}

The wave lengths given in Table I were compared with all series of grating measurement of the iron spectrum which have been made on the I. A. system. The results of this comparison are given for the benefit of anyone who may wish to use the grating determinations to fill in gaps in the series determined by means of the interferometer. The recent measurements of Goos, ${ }^{12}$ $3370 \mathrm{~A}$ to $4233 \mathrm{~A}$, are very consistent with the interferometer values, the mean difference Goos minus B. S. being o.oor $7 \mathrm{~A}$. The older work of Goos ${ }^{13}$ was done with a different instrument and the agreement with Table $\mathrm{I}$ is not good to the red of $4400 \mathrm{~A}$. The work of Janicki, ${ }^{14} 4282 \mathrm{~A}$ to $4903 \mathrm{~A}$, is in excellent agreement with the B. S. values, the mean difference Janicki minus B. S. being $0.0015 \mathrm{~A}$. Aside from the lines for which the values observed on Mount Wilson differ from those found at Pasadena, the wave lengths of St. John and Ware ${ }^{15}$ (4127A to $\left.6494 \mathrm{~A}\right)$ are also in

\footnotetext{
${ }^{11}$ Goos, Zs. f. wiss. Phot., 12, p. 259; I9r3.

12 Goos, Astron. Nach., 199, p. 3 ; igr4.

13 Goos, Zs. f. wiss. Phot., 11, pp. I, 305; 1912.

14 Janicki, Zs. f. wiss. Phot., 13, I73; 1913.

${ }_{15}$ St. John and Ware, Astroph. J., 36, p. 14, 1912; 38, p. 5, 1913.
} 
close agreement with the Bureau results. The mean difference St. John and Ware minus B. S. is about o.oor6A. The work of Viefhaus ${ }^{16}$ is not in agreement with the $B$. S. results for wave lengths shorter than $3800 \mathrm{~A}$, and not in very good agreement for the lines of greater wave length. The tables of Burns ${ }^{17}$ extend throughout the entire region under consideration and these values are in fair agreement with the B. S. system throughout, the mean difference Burns minus B. S. being o.oorg.

The differences, Grating minus B. S., were taken for each series and these data were collected for each $200 \mathrm{~A}$. In 5 out of these $\mathrm{I} 3$ regions the algebraic means for the best grating measurements agreed in showing a difference between the B. S. and the grating scale. This is no doubt due in part to an accumulation of errors of like sign in the B. S. table, and in part to the fact that the grating follows the local standards much more closely than does the interferometer. Further, the grating observers used some manganese standards and some iron standards which were too poor to be observed on our plates. The greatest difference is $0.0019 \mathrm{~A}$ in the region $6200 \mathrm{~A}$ to $6400 \mathrm{~A}$.

The arc has been used under normal conditions in only a comparatively small part of the grating work that has been done to date, and eventually the work will be repeated, observing under normal conditions. Further, there will eventually be other interference measurements of the greater part of the lines contained in Table I, and the means will be taken to establish I. A. standards. For these reasons the correction of the grating observations so as to make them fit the B. S. scale does not seem to be justified at present. And, for about one-third of the spectrum, the mean of the grating observations without correction would not make the individual lines agree much better with the interferometer work. If it is desired to fill a gap in Table I with a wave length which is fairly consistent with the B. S. scale, it seems best at present, all things considered, to take the value from any one of the grating series for which the mean value of Grating minus B. S. is less than $0.002 \mathrm{~A}$.

\section{INTENSITY EQUATION}

By this is meant any difference in wave length which is a function of the intensity of the line. It is illustrated by the differences St. John and Ware minus Burns in the region $5200 \mathrm{~A}$ to 
$5400 \mathrm{~A}$. The mean difference of six lines of intensity two and three is $+0.0035 \mathrm{~A}$, all differences being plus; the mean for eight lines of intensity four and five is -0.0004 , the differences being zero, positive, and negative; finally, the mean difference for six lines of intensity greater than five is +0.0002 , the differences again being zero, positive, and negative. It is plain that in this region the wave lengths of faint lines are systematically greater as measured by St. John and Ware than as measured by Burns. This effect is often seen in the comparison of the results from two grating plates of long exposure, particularly if there has been a change of temperature during either exposure. It may also result from slight errors in the adjustment of the grating, or from the use of an imperfect grating. If such an equation is found, only lines of the same intensity as the standards can be measured with the highest precision. Consequently the most careful examination for this phenomenon was made in connection with the interference observations. Only one plate showed any certain evidence of it.

On the other hand, comparison with the best series of grating observations showed this effect in every case. That the fault did not lie with the interferometer seems evident from the fact that in only one limited extent of spectrum were the different series of grating observations all in accord. The differences, Grating minus B. S., were collected into three groups with reference to the intensity of the lines; faint, moderate, and strong. Then the mean difference for the moderate lines was subtracted from that for the faint lines, and the mean difference for the strong lines was subtracted from that for the moderate lines. For one excellent series of observations this procedure gave positive results throughout the whole spectrum; that is, the grating obtained a longer wave length for the faint lines. In the case of another series of grating observations the opposite was true for the greater part of the spectrum. Thus, the comparison with grating measurements tends to strengthen the view that the interferometer wave lengths are comparatively unaffected by any intensity equation. 


\section{CHARACTER OF CERTAIN ADDITIONAL LINES}

\section{REJECTED INTERFERENCE OBSERVATIONS}

The following lines, formerly observed by one of us ${ }^{18}$ by the interference method, were omitted from Table I for various reasons. In Table 3 " $\mathrm{p}$ " indicates that the values determined from individual plates were too discordant; "1. w.," low weight, indicates insufficient observations; "d" means double; " $\mathrm{f}$ " means faint; and " $\mathrm{b}$ " indicates that the rings lacked sharpness.

TABLE 3

Rejected Interference Observations

\begin{tabular}{c|c||c|c|c|c}
\hline Wave length & Notes & Wave length & Notes & Wave length & Notes \\
\hline 3443 & d & 5602 & $(a)$ & 5984 & b, p \\
3468 & d & 5712 & f & 6020 & $(a)$ \\
5436 & f, p & 5714 & f & 6358 & $($ b $)$ \\
5483 & b, p & 5809 & p & 6569 & b, 1. w. \\
5567 & f, p & 5952 & p & & \\
5598 & b, l. w. & 5956 & p & & \\
\hline
\end{tabular}

a There is another line too close to 5602 and 6020 .

$b$ The poor agreement from one interferometer to another suggests a satellite in the case of 6358 .

\section{TERTIARY STANDARDS}

In Table 4 is found a list of those lines which have been suggested for tertiary standards by two or more investigators, and which have not been observed by interference. Column I contains the wave length as measured by Burns. ${ }^{19}$ For reasons stated above, the wave lengths were selected from one of the series of grating observations, and that of Burns was chosen on account of its being the only one which includes all of the lines. Column 2 gives the intensity and character of each line; $\mathrm{R}=$ broad reversal; for other symbols see description of Table $\mathrm{I}$. Column 3 indicates the order of interference in thousands which these lines show on the Bureau plates. As in Table I, the asterisk (*) indicates that the rings were poor, and the exclamation point (!) shows lack of interference. With rare exceptions, these lines are less sharp, and therefore, no doubt, less suitable for standards, than other lines in their neighborhood which have already been measured by interference methods. 
TABLE 4

Character of Certain Suggested Standards

\begin{tabular}{|c|c|c|c|c|c|c|c|c|c|c|c|}
\hline \multirow{2}{*}{$\begin{array}{r}\text { Wave lengths } \\
3372.081 \\
78.682 \\
87.410 \\
3406.807 \\
10.175\end{array}$} & \multirow{2}{*}{$\begin{array}{l}\text { I } \\
3 \\
4 \\
4 \\
4 \\
2\end{array}$} & \multicolumn{4}{|c|}{ Order } & \multirow{2}{*}{\begin{tabular}{r|} 
Wave lengths \\
95.004 \\
97.516 \\
98.512 \\
99.548 \\
3806.702
\end{tabular}} & \multirow{2}{*}{$\begin{array}{l}I \\
6 r \\
5 \\
6 r \\
6 r \\
6\end{array}$} & \multicolumn{4}{|c|}{ Order } \\
\hline & & $\begin{array}{l}22 \\
22 \\
22 \\
22 \\
22\end{array}$ & $\begin{array}{l}30 \\
30 \\
30 \\
29 \\
29\end{array}$ & $\begin{array}{l}44^{*} \\
44 \\
44 \\
44\end{array}$ & & & & $\begin{array}{l}11^{*} \\
20 \\
11^{*} \\
11^{*} \\
20\end{array}$ & $\begin{array}{l}20 ! \\
26 \\
20 * \\
20 ! \\
26\end{array}$ & $\begin{array}{l}40 \\
261 \\
39\end{array}$ & $53 *$ \\
\hline $\begin{array}{l}25.021 \\
31.827 \\
37.958 \\
40.614 \\
40.992\end{array}$ & $\begin{array}{l}4 \\
2 \mathrm{~b} \\
2 \\
7 \mathrm{R} \\
6 \mathrm{R}\end{array}$ & $\begin{array}{l}22 \\
22 \\
22 \\
12 ! \\
12 !\end{array}$ & $\begin{array}{l}29 \\
29 \\
29\end{array}$ & 44 & & $\begin{array}{l}08.732 \\
15.844 \\
20.430 \\
25.886 \\
27.826\end{array}$ & $\begin{array}{l}2 \\
7 R \\
8 R \\
8 R \\
6 R\end{array}$ & $\begin{array}{l}20 \\
11 ! \\
10 ! \\
10 ! \\
10 !\end{array}$ & 26 & 39 & 53 \\
\hline $\begin{array}{l}51.920 \\
52.279 \\
65.864 \\
69.012 \\
69.834\end{array}$ & $\begin{array}{l}6 \\
4 \\
6 \mathrm{R} \\
2 \\
2\end{array}$ & $\begin{array}{l}22 \\
22 \\
22 \\
22\end{array}$ & $\begin{array}{l}29 \\
29 \\
12 ! \\
29 \\
29\end{array}$ & $\begin{array}{l}43 \\
43\end{array}$ & & $\begin{array}{l}34.227 \\
40.443 \\
41.052 \\
46.806 \\
56.373\end{array}$ & $\begin{array}{l}7 \mathbf{R} \\
6 \mathbf{R} \\
6 \mathbf{R} \\
5 \\
6 \mathbf{R}\end{array}$ & $\begin{array}{l}10 ! \\
10 ! \\
10 ! \\
20 \\
10 !\end{array}$ & 26 & 39 & $52^{*}$ \\
\hline $\begin{array}{l}74.436 \\
77.850 \\
78.623 \\
83.012 \\
90.577\end{array}$ & $\begin{array}{l}2 \\
2 \\
2 \\
4 \\
6 \mathrm{R}\end{array}$ & $\begin{array}{l}22 \\
22 \\
22 \\
21 \\
12 !\end{array}$ & $\begin{array}{l}29 \\
29 \\
29 \\
29\end{array}$ & $43 !$ & & $\begin{array}{l}59.913 \\
65.526 \\
72.506 \\
78.024 \\
83.288\end{array}$ & $\begin{array}{l}7 \mathrm{R} \\
6 \mathrm{r} \\
6 \mathrm{r} \\
6 \mathrm{r} \\
2 .\end{array}$ & $\begin{array}{l}10 ! \\
10^{*} \\
10^{*} \\
10^{*} \\
19\end{array}$ & $\begin{array}{l}19 * \\
19 * \\
19 * \\
26\end{array}$ & $\begin{array}{l}26 ! \\
26 ! \\
26 ! \\
39\end{array}$ & 51 \\
\hline $\begin{array}{r}3500.570 \\
04.866 \\
05.065 \\
08.492 \\
10.443\end{array}$ & $\begin{array}{l}2 \\
2 \\
2 \\
4 \mathrm{v}, \mathrm{d} \\
2\end{array}$ & $\begin{array}{l}21 \\
21 \\
21 \\
21 \\
21\end{array}$ & $\begin{array}{l}28 \\
28 \\
28 \\
28 * \\
28\end{array}$ & $\begin{array}{l}43 \\
43 ! \\
43 \\
43 ! \\
43^{*}\end{array}$ & & $\begin{array}{l}86.287 \\
88.520 \\
95.659 \\
97.892 \\
98.013\end{array}$ & $\begin{array}{l}7 \mathrm{R} \\
7 \mathrm{~b} \\
5 \mathrm{r} \\
4 \\
4\end{array}$ & $\begin{array}{l}10 ! \\
19 * \\
10 * \\
19 \\
19\end{array}$ & $\begin{array}{l}26 ! \\
19 ! \\
26 \\
26\end{array}$ & $\begin{array}{l}38 \\
38\end{array}$ & $\begin{array}{l}51^{*} \\
51^{*}\end{array}$ \\
\hline $\begin{array}{l}33.004 \\
33.196 \\
49.873 \\
52.840 \\
67.045\end{array}$ & $\begin{array}{l}4 \\
5 \\
3 \\
4 \mathrm{~h} \\
4 \mathrm{~h}\end{array}$ & $\begin{array}{l}21 \\
21 \\
21 \\
21 \\
21\end{array}$ & $\begin{array}{l}28 \\
28 \\
28 \\
28^{*} \\
28\end{array}$ & $\begin{array}{l}43^{*} \\
43^{*} \\
42 \\
42 ! \\
42 !\end{array}$ & $56 *$ & $\begin{array}{r}99.711 \\
3902.950 \\
06.484 \\
13.635 \\
20.261\end{array}$ & $\begin{array}{l}6 r \\
7 r \\
5 r \\
2 \\
6 r\end{array}$ & $\begin{array}{l}10 * \\
10 * \\
10 * \\
19 \\
10^{*}\end{array}$ & $\begin{array}{l}19 ! \\
19 ! \\
19 * \\
26 \\
19 !\end{array}$ & $\begin{array}{l}26 * \\
38\end{array}$ & 51 \\
\hline $\begin{array}{l}70.102 \\
81.197 \\
82.202 \\
87.749 \\
89.453\end{array}$ & $\begin{array}{l}7 \mathrm{R} \\
8 \mathrm{R} \\
4 \\
2 \mathrm{~b} \\
3\end{array}$ & $\begin{array}{l}11 ! \\
11 ! \\
21 \\
21 \\
21\end{array}$ & $\begin{array}{l}28 \\
28 \\
28\end{array}$ & $\begin{array}{l}42 \\
42 ! \\
42\end{array}$ & $\begin{array}{l}56^{*} \\
56^{*}\end{array}$ & $\begin{array}{l}22.917 \\
25.650 \\
30.304 \\
32.635 \\
42.446\end{array}$ & $\begin{array}{l}6 \mathrm{R} \\
2 \\
7 \mathrm{R} \\
3 \\
3\end{array}$ & $\begin{array}{l}10 ! \\
19 \\
10 ! \\
19 \\
19\end{array}$ & $\begin{array}{l}26 \\
25 \\
25\end{array}$ & $\begin{array}{l}38 \\
38 \\
38\end{array}$ & $\begin{array}{l}51 \\
51 \\
51\end{array}$ \\
\hline $\begin{array}{r}99.628 \\
3602.515 \\
08.860 \\
31.464 \\
47.845\end{array}$ & $\begin{array}{l}3 \\
2 \mathrm{v} \\
6 \mathrm{R} \\
6 \mathrm{R} \\
6 \mathrm{R}\end{array}$ & $\begin{array}{l}21 \\
21 * \\
11 ! \\
11 ! \\
11 !\end{array}$ & $\begin{array}{l}28 \\
28 !\end{array}$ & 42 & $56 !$ & $\begin{array}{l}49.956 \\
51.165 \\
56.461 \\
56.682 \\
67.426\end{array}$ & $\begin{array}{l}4 \\
4 \\
4 \\
6 \\
4\end{array}$ & $\begin{array}{l}19 \\
19 \\
19 \\
19 \\
19\end{array}$ & $\begin{array}{l}25 \\
25 \\
25 \\
25 \\
25\end{array}$ & $\begin{array}{l}38 \\
38 \\
38 \\
38 \\
38\end{array}$ & $\begin{array}{l}51 \\
51 \\
51 \\
51 \\
50\end{array}$ \\
\hline $\begin{array}{l}55.470 \\
67.280 \\
74.768 \\
78.862 \\
85.995\end{array}$ & $\begin{array}{l}4 \\
4 h \\
2 \\
2 \\
5 h\end{array}$ & $\begin{array}{l}21 \\
20 \\
20 \\
20 \\
20\end{array}$ & $\begin{array}{l}27 \\
27 * \\
27 \\
27 \\
27^{*}\end{array}$ & $\begin{array}{l}41 \\
41 ! \\
41 \\
41 \\
41 !\end{array}$ & $\begin{array}{l}55^{*} \\
54^{*} \\
54\end{array}$ & $\begin{array}{l}69.263 \\
71.328 \\
81.776 \\
86.178 \\
95.989\end{array}$ & $\begin{array}{l}7 \mathrm{r} \\
4 \\
3 \\
3 \\
1\end{array}$ & $\begin{array}{l}10 ! \\
19 \\
19 \\
19 \\
19\end{array}$ & $\begin{array}{l}25 \\
25 \\
25 \\
25\end{array}$ & $\begin{array}{l}38 \\
38 \\
38 \\
38\end{array}$ & $\begin{array}{l}50 \\
50 \\
50 \\
50\end{array}$ \\
\hline $\begin{array}{r}97.436 \\
3711.227 \\
11.408 \\
19.938 \\
22.565\end{array}$ & $\begin{array}{l}2 \mathrm{~h} \\
2 \\
1 \\
8 \mathrm{R} \\
6 \mathrm{R}\end{array}$ & $\begin{array}{l}20 \\
20 \\
20 \\
11 ! \\
11 !\end{array}$ & $\begin{array}{l}27 * \\
27 \\
27\end{array}$ & $\begin{array}{l}41 ! \\
40 \\
40\end{array}$ & $\begin{array}{l}54 \\
54\end{array}$ & $\begin{array}{r}98.059 \\
4005.250 \\
07.233 \\
17.154 \\
24.745\end{array}$ & $\begin{array}{l}5 \\
7 \mathrm{~b} \\
1 \\
3 \\
2\end{array}$ & $\begin{array}{l}19 \\
10^{*} \\
19 \\
19 \\
19\end{array}$ & $\begin{array}{l}25 \\
19^{*} \\
25 \\
25 \\
25^{*}\end{array}$ & $\begin{array}{l}38 \\
25 ! \\
37 \\
37 \\
37 !\end{array}$ & $\begin{array}{l}50 \\
50 \\
50\end{array}$ \\
\hline $\begin{array}{l}26.922 \\
27.095 \\
30.390 \\
34.869 \\
37.135\end{array}$ & $\begin{array}{l}3 \mathbf{h} \\
2 \\
3 \\
9 \mathbf{R} \\
7 \mathbf{R}\end{array}$ & $\begin{array}{l}20 \\
20 \\
20 \\
11 ! \\
11 !\end{array}$ & $\begin{array}{l}27 \\
27 \\
27\end{array}$ & $\begin{array}{l}40 ! \\
40 ! \\
40\end{array}$ & $53^{*}$ & $\begin{array}{l}32.636 \\
45.822 \\
63.604 \\
67.279 \\
76.641\end{array}$ & $\begin{array}{l}1 \\
8 R \\
8 R \\
3 \\
5\end{array}$ & $\begin{array}{l}19 \\
10 ! \\
10 ! \\
18 \\
18\end{array}$ & $\begin{array}{l}25 \\
24^{*}\end{array}$ & $\begin{array}{l}37 \\
37 !\end{array}$ & 49 \\
\hline $\begin{array}{r}3742.623 \\
46.927 \\
48.264 \\
49.487 \\
58.234\end{array}$ & $\begin{array}{l}1 \mathbf{h} \\
2 \mathrm{~h} \\
6 \mathrm{R} \\
8 \mathrm{R} \\
7 \mathrm{R}\end{array}$ & $\begin{array}{l}20 \\
20 \\
11 ! \\
11 ! \\
11 !\end{array}$ & $\begin{array}{l}27 \\
27 *\end{array}$ & $\begin{array}{l}40^{*} \\
40 !\end{array}$ & $53 !$ & $\begin{array}{r}78.362 \\
79.847 \\
84.508 \\
98.189 \\
4109.810\end{array}$ & $\begin{array}{l}3 \\
2 \\
4 \\
3 \\
4\end{array}$ & $\begin{array}{l}18 \\
18 \\
18 \\
18 \\
18\end{array}$ & $\begin{array}{l}24 \\
24 \\
24 \\
24 \\
24\end{array}$ & $\begin{array}{l}37 \\
37 \\
37 \\
37 ! \\
37\end{array}$ & $\begin{array}{l}49 \\
49 \\
49 ! \\
49\end{array}$ \\
\hline $\begin{array}{l}63.792 \\
67.194 \\
76.456 \\
87.880 \\
90.094\end{array}$ & $\begin{array}{l}6 \mathrm{R} \\
6 \mathrm{R} \\
2 \\
6 \mathrm{R} \\
4\end{array}$ & $\begin{array}{l}11 ! \\
11 ! \\
20 \\
11 ! \\
20\end{array}$ & $\begin{array}{l}26 \\
26\end{array}$ & $\begin{array}{l}40 \\
40\end{array}$ & $\begin{array}{l}53 \\
53 !\end{array}$ & $\begin{array}{l}14.454 \\
21.809 \\
32.064 \\
37.002 \\
43.874\end{array}$ & $\begin{array}{l}4 \\
2 \\
7 \\
3 \\
7\end{array}$ & & $\begin{array}{l}24 \\
24 \\
24 * \\
24 \\
24 *\end{array}$ & $\begin{array}{l}37 \\
36 \\
36 ! \\
36 \\
36 !\end{array}$ & $\begin{array}{l}49 \\
49 \\
48\end{array}$ \\
\hline
\end{tabular}


TABLE 4-Continued

Character of Certain Suggested Standards-Continued

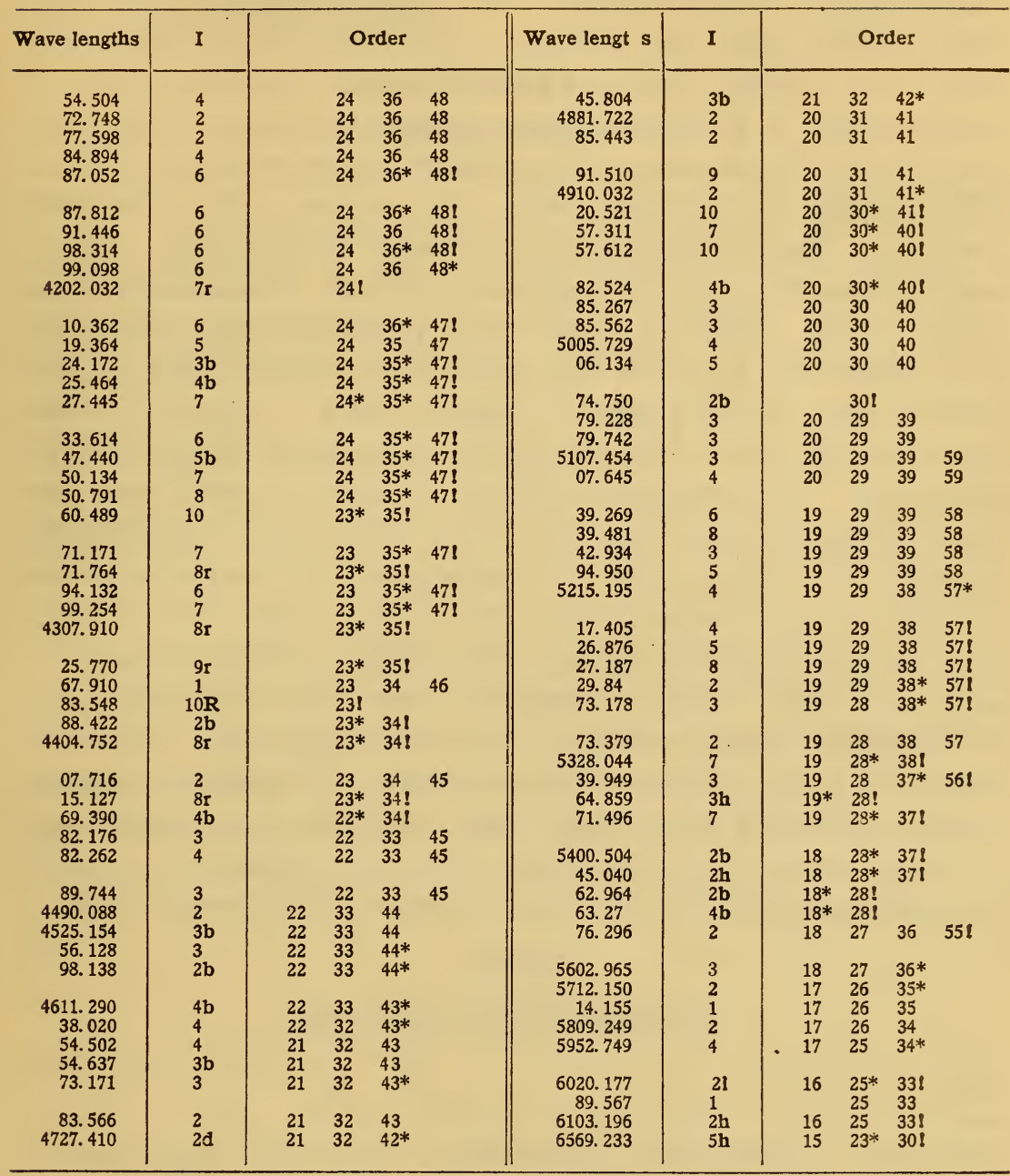

\section{CORRELATION OF SHARPNESS WITH OTHER PROPERTIES}

\section{INTRODUCTION}

Five hundred and thirty of the lines contained in Tables I and 4 were examined at various orders of interference and were either found to be sharp enough to stand a high order of interference, or their limiting orders were determined. Forty-nine additional lines, all of which are easily reversed, were found to show no inter$50239^{\circ}-16-7$ 
ference, even at an order of about ro ooo. These lines and about 50 others for which the data were incomplete do not enter into the discussion which follows. The lines examined were arbitrarily divided into four groups. In the first group interference ceased to be visible when the path difference was from 20000 to 30 ooo waves; in the second group, 30000 to 40000 ; in the third group, 40000 to 60000 ; and the fourth group contains the lines still showing interference with the largest path differences observed. The last group is not entirely homogeneous. All the lines in this group show interference with an order of more than 40000 waves. Some of them would probably have been found to be limited by 60000 if they had been examined that far, but most of them would possess a limiting order in excess of 60000 waves because they still show perfect interference between 40000 and 60000 . The groups are distinct enough to justify some general conclusions when correlated with other data. The material contained in Tables $\mathrm{I}$ and 4 is incomplete, but so far as it goes it points to the conclusion that the limiting order of the average iron line is independent of the wave length. Fundamentaily, we have to do with vibration frequency, and the above shows that the variation from the mean is the same proportion of the frequency for lines in all parts of the spectrum. Or putting it another way, the width of the average line, expressed as a proportion of the wave length, is constant throughout the spectrum; expressed in angstroms, the width is a direct function of the wave length.

TABLE 5

Percentage of Lines in Each Group

\begin{tabular}{l|c|c|c|c}
\hline Group.............. & I & II & III & IV \\
\hline Limiting order.... & 20000 to 30000 & 30000 to 40000 & 40000 to 60000 & $\begin{array}{c}\text { Above the largest ob- } \\
\text { served }\end{array}$ \\
\hline $\begin{array}{l}\text { Number of lines.... } \\
\begin{array}{l}\text { Per cent of total } \\
\text { lines }\end{array}\end{array}$ & 39 & 48 & 102 \\
19 & 341 \\
\hline
\end{tabular}

\section{POLE EFFECT}

A correlation of these data with those of St. John and Babcock $^{20}$ on "Pole effect" is represented in Table 6. Pole effect indicates a change in wave length when the light is taken from the negative pole instead of the center of the arc. Positive 
shifts indicate increased wave lengths from the negative pole as compared with the center and negative shifts are displacements toward shorter wave lengths.

TABLE 6

Sharpness and Pole Effect

\begin{tabular}{|c|c|c|c|c|c|}
\hline Group............................... & I & II & III & IV & Totals \\
\hline Number of positive shifts... & 6 & 25 & 41 & 35 & 107 \\
\hline Per cent of positive shifts.... & 6 & 22 & 39 & 33 & 100 \\
\hline Number of negative shifts........ & 12 & 6 & 6 & 0 & 24 \\
\hline Per cent of negative shifts....................... & 50 & 25 & 25 & 0 & 100 \\
\hline Positive shifts $<.010 \mathrm{~A} \ldots \ldots \ldots \ldots \ldots$ & 4 & 6 & 3 & 1 & 14 \\
\hline 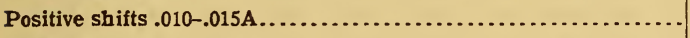 & 1 & 13 & 6 & 14 & 34 \\
\hline Positive shifts $.015-.020 \mathrm{~A} \ldots \ldots \ldots \ldots$ & 1 & 2 & 12 & 13 & 28 \\
\hline 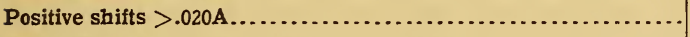 & 0 & 4 & 20 & 7 & 31 \\
\hline Negative shifts $<.010 \mathrm{~A} . \ldots \ldots \ldots$ & 1 & 3 & 4 & 0 & 8 \\
\hline Negative shifts $.010-.015 A \ldots \ldots \ldots \ldots \ldots \ldots \ldots . . . . . . .$. & 4 & 1 & 2 & 0 & 7 \\
\hline Negative shifts $.015-.020 \mathrm{~A} \ldots \ldots \ldots \ldots \ldots \ldots$ & 4 & 0 & 0 & 0 & 4 \\
\hline Negative shifts $>.020 \mathrm{~A} . \ldots \ldots \ldots \ldots \ldots \ldots$ & 3 & 2 & 0 & 0 & 5 \\
\hline
\end{tabular}

About ig per cent of the lines examined have positive shifts and a little over 4 per cent have negative shifts. It is interesting to observe that the majority of positive shifts occur with lines which are known to be sharp in the center of the arc because they show interference of rather a high order, while the negative shifts occur principally with poorer lines. Negative shifts are entirely absent from group IV and only a few small ones (less than o.or $5 \mathrm{~A}$ ) are found in group III. Positive shifts are mostly larger than o.oI $5 \mathrm{~A}$ and occur most frequently in groups III and IV.

Pole shifts of positive sign were obtained by St. John and Babcock from classes " $c 5$ " and " $d$ " of the Gale and Adams ${ }^{21}$ classification of lines. Large pressure displacements are indicated by "c" and "d," and " 5 " means unsymmetrical widening toward the red. Negative shifts affect lines which St. John and Ware ${ }^{22}$ have placed in a separate class, "e," because they are widened unsymmetrically toward the violet by pressure, and greatly displaced in this direction.

No satisfactory explanation of this pole effect can be given at the present time. Fortunately it may be excluded by using only the center of the arc, as recommended by the international committee. 


\section{INTENSITY}

An attempt was made to connect limiting order and pole effect with intensity. The data are arranged in Table 7 .

\section{TABLE 7}

Sharpness, Intensity, and Pole Effect

\begin{tabular}{|c|c|c|c|c|c|c|c|c|c|c|}
\hline Intensity & 1 & 2 & 3 & 4 & 5 & 6 & 7 & 8 & 9 & 10 \\
\hline Number of lines .... & 10 & 103 & 94 & 130 & 92 & 60 & 21 & 16 & 1 & 3 \\
\hline Number of lines, Group I. ........ & 0 & 11 & 6 & 6 & 6 & 2 & 5 & 2 & 0 & 1 \\
\hline Number of lines, Group II......... & 1 & 11 & 8 & 6 & 2 & 8 & 9 & 1 & 1 & 2 \\
\hline Number of lines, Group III ..... & 0 & 13 & 15 & 24 & 18 & 21 & 2 & 9 & 0 & 0 \\
\hline Number of lines, Group IV........ & 9 & 68 & 65 & 94 & 66 & 39 & 5 & 4 & 0 & 0 \\
\hline Per cent of lines, Group IV... & 90 & 66 & 69 & 72 & 71 & 48 & 24 & 25 & 0 & 0 \\
\hline Number of pole shifts ............ & 0 & 23 & 22 & 28 & 23 & 13 & 7 & 6 & 1 & 3 \\
\hline Per cent of lines affected ... & 0 & 22 & 23 & 22 & 25 & 22 & 33 & 38 & 100 & 100 \\
\hline Number of positive shifts .......... & 0 & 15 & 14 & 24 & 21 & 12 & 7 & 5 & 1 & 3 \\
\hline Number of negative shifts ....... & 0 & 8 & 8 & 4 & 2 & 1 & 0 & 1 & 0 & 0 \\
\hline
\end{tabular}

Broadly speaking, it is seen that limiting order is an inverse function of intensity, and that the frequency of positive shifts tends to increase with large intensities. About 68 per cent of the. lines included by intensities $I$ to 6 still show interference of an order greater than 40000 , against only 22 per cent of the lines of greater intensity. On the other hand, only 24 per cent of the lines of intensity I to 6 , inclusive, have pole shifts against $4 \mathrm{I}$ per cent of the more intense lines. Lines of small intensity are more frequently affected by negative pole shifts, taking into account only lines in this list.

St. John and Babcock examined I570 iron lines between 2979 and 6678 and found 23 per cent of the lines affected by pole effect. Among our 530 lines there are I3 I affected lines, or about 24 per cent, so that the lines chosen or suggested as standards appear to be representative as far as pole effect is concerned. Inasmuch as practically all the lines in our lists were examined for pole effect, the conclusions drawn from these tables will probably be valid generalizations.

\section{BEHAVIOR UNDER PRESSURE}

The data on limiting order were also correlated as far as possible with the Gale and Adams classification of lines, and the results are found in Table 8. 
TABLE 8

Sharpness and Pressure Effect

\begin{tabular}{|c|c|c|c|c|c|}
\hline 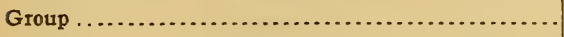 & I & II & III & IV & Total \\
\hline Class 1a... & & 3 & 1 & & 4 \\
\hline Class $3 a . . . .$. & & & & 8 & 8 \\
\hline Class $4 a . . . . . . . . . .$. & & . & 9 & 3 & 12 \\
\hline 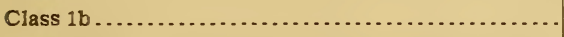 & 3 & 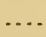 & 1 & 1 & 5 \\
\hline Class $2 \mathrm{~b} . . .$. & & 2 & & & 2 \\
\hline Class $3 \mathrm{~b} . . . \ldots \ldots \ldots$ & & ... & $\cdots$ & 9 & 9 \\
\hline Class $4 \mathrm{~b} . . . . . . . .$. & & & & 27 & 27 \\
\hline 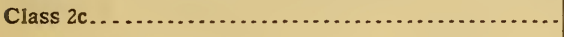 & 1 & & & 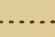 & 1 \\
\hline 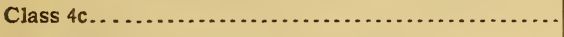 & & 1 & & 7 & 8 \\
\hline 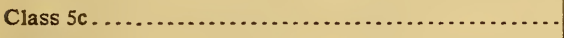 & & 4 & $\cdots$ & 7 & 11 \\
\hline Class $5 d, \ldots \ldots \ldots \ldots$ & $\cdots \cdot$ & 4 & 14 & 3 & 21 \\
\hline Class e...$\ldots \ldots \ldots \ldots \ldots \ldots \ldots$ & 12 & 6 & 6 & & 24 \\
\hline
\end{tabular}

Gale and Adams ${ }^{23}$ divide spectrum lines into five broad classes, according to their behavior under pressure, as follows:

I. Lines which are symmetrically reversed.

2. Lines which are unsymmetrically reversed.

3. Lines which remain bright and fairly narrow under pressure.

4. Lines which remain bright and symmetrical but become wide and diffuse under pressure.

5. Lines which remain bright and are widened very unsymmetrically toward the red.

Measures of the displacements of iron lines under pressure led Gale and Adams to form four groups of lines represented by letters a, b, c, and d. Group a includes all flame lines and these show only small displacements. Group $b$ is a large one and includes all lines of small displacements which are not included in group a. Group c consists of lines showing much larger displacements than those of group b. Group $d$ is made up of several scattered groups, all of which show immense displacements. If all displacement values are reduced to that of group a as the unit, the following relation exists: ra, r.5b, 3.4c, and $6.6 \mathrm{~d}$.

The table shows that lines in classes $3 \mathrm{a}, 3 \mathrm{~b}, 4 \mathrm{~b}$, and $4 \mathrm{c}$ are capable of producing interference of the highest order observed, and these lines recommend themselves for use as standards. The majority of $4 \mathrm{a}$ and $5 \mathrm{~d}$ lines examined reach their limiting order of interference between 40000 and 60000 waves. 


\section{SUMMARY}

Between the limits $3233 \mathrm{~A}$ and $6750 \mathrm{~A}$, some 400 iron lines were either remeasured or measured for the first time by the interference method. The work was done in conformity with the recommendation of the international wave length committee. Where it was found possible, faint lines as well as strong ones were measured. Satisfactory standards were found at intervals of Io or 15 angstroms throughout the greater part of the spectrum; there are no sharp iron lines of even moderate intensity in the region $5775 \mathrm{~A}$ to $5934 \mathrm{~A}$, and the number is insufficient in the extreme red.

The spectroscope used was a $635 \mathrm{~cm}$ concave grating, mounted in parallel light. The greater part of the wave lengths were measured by means of three interferometers, the order of interference ranging from 20000 to 50000 in the case of each line. The method was that of Buisson and Fabry.

The mean difference between the present observations and the I. A. standards is about one part in four million. It is thought that some of the standards are in error by $0.002 \mathrm{~A}$ or more.

Interferometer observations under the conditions described were found to be reasonably free from the effects of pole shifts.

Comparison with all the grating observations which have been made on the I. A. system shows, as has been found before, that more numerous secondary standards are needed in order to obtain the highest accuracy in grating work.

While grating observations often show an intensity equation, interference measurements appear to be reasonably free from this effect.

The sharpness of over 600 lines was determined and the data were correlated with data relating to pole shifts, to behavior under pressure, and to intensity. Lines showing a negative pole shift are never very sharp, while those having a positive pole shift are sometimes perfectly sharp in the center of the arc; the lines subject to a shift are more likely than are the unaffected lines to be broad, even in the center of the arc. Lines which are faint or of only moderate intensity are sharper on the average than strong lines. Still, some very strong lines are quite sharp.

The width of the average line, expressed as a proportion of the wave number, is not a function of the wave length.

Washington, December 2I, I9I 5 . 